\title{
Gauged lepton flavour
}

\section{R. Alonso, ${ }^{a, c}$ E. Fernandez Martinez, ${ }^{b}$ M.B. Gavela, ${ }^{b}$ B. Grinstein, ${ }^{a}$ L. Merlo ${ }^{b}$ and P. Quilez}

${ }^{a}$ Dept. of Physics, University of California, San Diego, 9500 Gilman Drive, La Jolla, CA, 92093-0319 U.S.A.

${ }^{b}$ Departamento de Física Teórica and Instituto de Física Teórica, IFT-UAM/CSIC, Universidad Autónoma de Madrid, C/ Nicolas Cabrera 13-15, Cantoblanco, Madrid, 28049 Spain ${ }^{c}$ CERN, Theory Division, Geneva 23, CH-1211 Switzerland

E-mail: rodrigo.alonso@cern.ch, enrique.fernandez-martinez@uam.es, belen.gavela@uam.es, bgrinstein@ucsd.edu, luca.merlo@uam.es, pablo.quilez@uam.es

ABstRACT: The gauging of the lepton flavour group is considered in the Standard Model context and in its extension with three right-handed neutrinos. The anomaly cancellation conditions lead to a Seesaw mechanism as underlying dynamics for all leptons; requiring in addition a phenomenologically viable setup leads to Majorana masses for the neutral sector: the type I Seesaw Lagrangian in the Standard Model case and the inverse Seesaw in the extended model. Within the minimal extension of the scalar sector, the Yukawa couplings are promoted to scalar fields in the bifundamental of the flavour group. The resulting low-energy Yukawa couplings are proportional to inverse powers of the vacuum expectation values of those scalars; the protection against flavour changing neutral currents differs from that of Minimal Flavour Violation. In all cases, the $\mu-\tau$ flavour sector exhibits rich and promising phenomenological signals.

Keywords: Beyond Standard Model, Gauge Symmetry, Neutrino Physics, Spontaneous Symmetry Breaking

ARXiv EPrint: 1609.05902 


\section{Contents}

1 Introduction 1

2 Gauged lepton flavour standard model: $\mathrm{SU}(3)_{\ell} \times \mathrm{SU}(3)_{E} \quad 4$

2.1 Spectrum 7

$\begin{array}{lll}2.2 & \text { Interactions } & 12\end{array}$

$\begin{array}{lll}2.3 & \text { Phenomenology } & 14\end{array}$

3 Gauged lepton flavour Seesaw model: $\mathrm{SU}(3)_{\ell} \times \mathrm{SU}(3)_{E} \times \mathrm{SO}(3)_{N} \quad 18$

3.1 Fermion spectrum and interactions: $\mathcal{Y}_{N} \gg \mu_{\mathrm{LN}}$ case 20

3.1.1 $\mathcal{Y}_{E}>\left\|\mathcal{Y}_{N}\right\|$ - vectorial flavour-preserving gauge bosons 23

$\begin{array}{lll}\text { 3.1.2 } & \mathcal{Y}_{N}>\left\|\mathcal{Y}_{E}\right\|-\text { LUV and subleading cLFV } & 25\end{array}$

$\begin{array}{lll}\text { 3.1.3 } \mathcal{Y}_{E} \sim\left\|\mathcal{Y}_{N}\right\|-\mathrm{LUV} \text { and } \mathrm{cLFV} & 27\end{array}$

4 Comparison with minimal lepton flavour violation, for $\mathcal{Y}_{N} \gg \mathcal{Y}_{E} \quad 28$

5 Conclusions 33

Dedicated to Giotto di Bondone and

Hieronymus Bosch

\section{Introduction}

The tantalizing pattern of masses and mixings of the elementary particles composing the visible universe calls for a change of paradigm. The origin of flavour lurks behind the limits of our understanding of the Standard Model (SM). Beyond the perplexity of "why three fermion generations with such diverse masses and mixings", the flavour puzzle is also central to attempts to solve SM fine-tunings. For instance, beyond the SM theories (BSM) attempting to solve the electroweak hierarchy problem typically convey unacceptable consequences in the flavour sector: this is known as the flavour problem. Flavour contributions are also one of the main ingredients in formulating the strong CP problem of the SM.

In the SM, the only source of flavour are the Yukawa couplings introduced as arbitrary numerical inputs, "just-so" numbers which account for the fermion masses and mixings. This consistent procedure is nevertheless unsatisfactory in its arbitrariness. Symmetries, and in particular gauged symmetries, have engendered our deepest understanding of particle dynamics and a decades-old unfulfilled dream is that of explaining the flavour puzzle in terms of a symmetry principle.

Attempts in this direction have been carried out in the past [1], generally from a topdown approach. A generic consequence of these models is that the explanation of the flavour puzzle is affected by the same flavour problem that afflicts many extensions of the 
SM, e.g., theories addressing the hierarchy problem. This issue of course does not disprove the models of flavour, but it does however place the scale of new physics well beyond direct probe [2].

Minimal flavour violation (MFV) $[3,4]$ is in contrast a bottom-up approach that aims at characterizing the low energy effects of a class models that are not afflicted by the flavour problem, e.g. SUSY models with gauge mediated SUSY breaking. The framework is based on the global flavour symmetry group that the SM exhibits in the limit of vanishing Yukawas [4], plus the simple assumption that at low-energies Yukawa couplings are the only source of flavour in the SM and in whatever the BSM theory of flavour is. For quarks, the flavour symmetry exhibited by the SM massless Lagrangian is [3]

$$
\mathrm{U}(3)_{Q} \times \mathrm{U}(3)_{u} \times \mathrm{U}(3)_{d},
$$

where $Q$ denotes quark $\mathrm{SU}(2)_{L}$ doublets and $u$ and $d$ stand for the right-handed components of up and down quarks. Yukawa couplings break the symmetry and they are then treated as spurions of the flavour group, weighting the possible BSM effective operators so as to make them invariant under the flavour group. As a consequence, MFV predicts the relative rates of flavour changing transitions, and furthermore new effects at or close the $\mathrm{TeV}$ scale are allowed.

The MFV ansatz is neither the only flavour ansatz compatible with data nor a theory of flavour, though. There have been attempts to go from the effective approach - where the Yukawas are treated as spurions - to a more fundamental level where the Yukawas are dynamical "flavon" fields, acquiring a non-trivial vacuum expectation value. The potentials for the corresponding scalar fields have been discussed for several possible flavour representations, with interesting consequences [1,5-14]. Although a dynamical justification for all fermion masses and mixings is still lacking, the potential minima lead for instance to no mixing at leading order in the quark sector (in contrast to the lepton sector discussed further below) when each Yukawa coupling is associated to a single flavon, a very encouraging first step.

Nevertheless, unless the continuous symmetry in eq. (1.1) is substituted by a convenient discrete subgroup, a generic consequence of breaking spontaneoulsy the SM global flavour group is that of unobserved goldstone bosons. Would instead the symmetry be gauged, the goldstone bosons would become the longitudinal degrees of freedom of massive vector bosons. This exploratory effort was launched for the quark sector in ref. [15] and continued in refs. [17-22]. In ref. [15] it was shown that the consistency of the gauge theory via anomaly cancellation conditions, requires the addition of fermions with drastic implications for phenomenology.

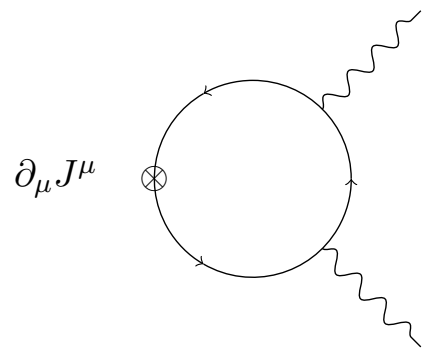


The masses of the extra fermionic content of those gauged-flavour models are inversely proportional to the masses of the light SM fermions (as it was introduced in ref. [16]), with the consequence that flavour-changing neutral currents (FCNC) are highly suppressed for light generations and new exotic gauge bosons could be as light as the electroweak scale. This theory, with gauge symmetry at its core, offers a different take on the number of generations; the fields must belong to irreducible representations of the flavour group and thus the number of generations is linked to it, in the precise same sense in which there are three colors in QCD. Although the starting motivation was the phenomenologically successful MFV ansatz, the mechanism for protection against the flavour problem in the gauged-flavour model does not conform to the MFV hypothesis; yet it is still very effective.

Here, the gauging of the lepton flavour group is considered. Our present knowledge of masses and mixing in this sector is summarized as [24, 25]:

$$
\begin{gathered}
m_{e}=0.511 \mathrm{MeV}, \quad m_{\mu}=0.106 \mathrm{GeV}, \quad m_{\tau}=1.78 \mathrm{GeV}, \\
\Delta m_{\mathrm{sol}}^{2}=\left(7.50_{-0.17}^{+0.19}\right) 10^{-5} \mathrm{eV}^{2}, \quad \Delta m_{\mathrm{atm}}^{2}=\left\{\begin{array}{ll}
(2.457 \pm 0.047) \times 10^{-3} \mathrm{eV}^{2} & \mathrm{NH} \\
\left(2.449_{-0.047}^{+0.048}\right) \times 10^{-3} \mathrm{eV}^{2} & \mathrm{IH}
\end{array},\right. \\
\theta_{12}=\left(33.48_{-0.75}^{+0.78}\right)^{\circ}, \quad \theta_{23}=\left\{\begin{array}{ll}
\left(42.3_{-1.6}^{+3.0}\right)^{\circ} & \mathrm{NH} \\
\left(49.5_{-2.2}^{+1.5}\right)^{\circ} & \mathrm{IH}
\end{array}, \theta_{13}=\left\{\begin{array}{ll}
\left(8.50_{-0.21}^{+0.20}\right)^{\circ} & \mathrm{NH} \\
\left(8.51_{-0.21}^{+0.20}\right)^{\circ} & \mathrm{IH}
\end{array},\right.\right.
\end{gathered}
$$

where only three significant digits and no errors have been reported for the charged lepton masses, as any further precision is below the present uncertainty on the other parameters.

In contrast to the quark case [15], the unknown nature of neutrino masses opens several possibilities for constructing a consistent model with the lepton flavour symmetry gauged, as evidenced by the various definitions of MFV in the lepton sector [26-30]. The guiding principle followed here will be to consider phenomenologically viable setups with:

- Maximal flavour symmetry group of the Lagrangian for massless SM fermions

- Minimal extension of the spectrum

In the absence of right-handed neutrinos and neglecting fermion masses, the SM leptonic Lagrangian is invariant under the continuous flavour group

$$
\mathrm{U}(3)_{\ell} \times \mathrm{U}(3)_{E},
$$

where $\ell$ denotes $\mathrm{SU}(2)_{L}$ leptonic doublets and the subscript $E$ stands for right-handed charged leptons. The cancellation of gauge anomalies of this pure SM case along the guidelines above will be shown to lead to the introduction of SM fermion singlets and thus to Majorana neutrinos as a very natural consequence.

If instead one assumes from the beginning the existence of three right-handed neutrino fields $\mathcal{N}_{R}$, two symmetry avenues are possible:

- Assuming Dirac neutrinos, the flavour group would be $\mathrm{U}(3)_{\ell} \times \mathrm{U}(3)_{E} \times \mathrm{U}(3)_{N}$, the subscript $N$ referring to the right-handed neutrinos [21]. 
- Assuming instead Majorana neutrinos, the maximal flavour group is $\mathrm{U}(3)_{\ell} \times \mathrm{U}(3)_{E} \times$ $O(3)_{N}$, leading naturally to a type I Seesaw [31-34] scenario with degenerate heavy neutrinos.

This last option has been shown $[10,11,13,14]$ to allow a minimum of its scalar potential with one maximal PMNS angle and Majorana phase (and a second angle generically large), at leading order and for minimal flavon content. In contrast, the $\mathrm{U}(3)^{3}$ case tends to disfavor large mixings, consistent with observations in the quark sector but in disagreement with the observed leptonic mixing. The guiding principles chosen above also favor the second option in that the extra field content needed is smaller, and therefore leads to more predictive models: this scenario will be thus analyzed in detail. Interestingly, both cases - that is with and without right-handed neutrinos - will lead to Majorana masses for the active neutrinos, so that at low energies the Lagrangian responsible for masses and mixings will be, for definiteness:

$$
\mathscr{L}_{Y}=-\bar{\ell}_{L} H Y_{E} e_{R}-\frac{1}{2} \bar{\ell}_{L} \widetilde{H} \frac{C_{\nu}}{\Lambda_{\mathrm{LN}}} \widetilde{H}^{T} \ell_{L}^{c}+\text { h.c. },
$$

where $H$ denotes the Higgs doublet, $\widetilde{H} \equiv i \sigma_{2} H^{*}, Y_{E}$ is the matrix of charged lepton Yukawa couplings, $\Lambda_{\mathrm{LN}}$ the generic scale of Lepton Number (LN) violation and $C_{\nu}$ the dimensionless coefficient of the Weinberg operator [35] which describes light neutrino masses. The leptonic mass matrices will then be given by

$$
m_{\ell}=Y_{E} \frac{v}{\sqrt{2}}, \quad m_{\nu}=\frac{v^{2}}{2} \frac{C_{\nu}}{\Lambda_{\mathrm{LN}}},
$$

where $v$ denotes the vacuum expectation value (vev) of the Higgs field, $v=246 \mathrm{GeV}$. The generalized Seesaw pattern obtained below, together with the lightness of the electron as compared to the $\tau$ and $\mu$ leptons, implies that the least broken subgroups of the flavour symmetry are expected to reside in the $\mu-\tau$ sector. The corresponding approximate symmetries, the spectrum of new particles and the dominant experimental signals will be determined and discussed in the following sections. Furthermore, the differences between the effective low-energy couplings of the gauged-flavour theory and the leptonic MFV ansatz will also be discussed.

The analysis will be restricted to the non-abelian sector of the global flavour symmetry, as the focus is set on flavour-changing effects; some phenomenological differences which result when gauging in addition the two non-anomalous abelian symmetries will be pointed out, though.

The structure of the paper can be easily inferred from the table of Contents.

\section{Gauged lepton flavour standard model: $\mathrm{SU}(3)_{\ell} \times \mathrm{SU}(3)_{E}$}

It will be shown in this section how the gauging of the pure SM leptonic flavour group favours a Seesaw pattern and Majorana neutrino masses, and that the leading phenomenological signals are lepton universality violation (LUV), with deviations from the SM predictions which are particularly prominent in the $\tau$ sector. 


\begin{tabular}{|c|cccc|}
\hline & $\mathrm{SU}(2)_{L}$ & $\mathrm{U}(1)_{Y}$ & $\mathrm{SU}(3)_{\ell}$ & $\mathrm{SU}(3)_{E}$ \\
\hline$\ell_{L} \equiv\left(\nu_{L}, e_{L}\right)$ & 2 & $-1 / 2$ & 3 & 1 \\
$e_{R}$ & 1 & -1 & 1 & 3 \\
\hline $\mathcal{E}_{R}$ & 1 & -1 & 3 & 1 \\
$\mathcal{E}_{L}$ & 1 & -1 & 1 & 3 \\
$\mathcal{N}_{R}$ & 1 & 0 & 3 & 1 \\
\hline $\mathcal{Y}_{E}$ & 1 & 0 & $\overline{3}$ & 3 \\
$\mathcal{Y}_{N}$ & 1 & 0 & $\overline{6}$ & 1 \\
\hline
\end{tabular}

Table 1. Transformation properties of SM fields, of (flavour) mirror fields and of flavons under the EW group and $\mathrm{SU}(3)_{\ell} \times \mathrm{SU}(3)_{E}$.

The leptonic global flavour symmetry to be gauged is that exhibited by the SM in the absence of Yukawa couplings, which is that of the kinetic terms,

$$
\mathscr{L}_{\text {leptons }}=i \bar{\ell}_{L} \not D \ell_{L}+i \bar{e}_{R} \not D e_{R} .
$$

Anomaly cancellation of the non-abelian $\mathrm{SU}(3)_{\ell} \times \mathrm{SU}(3)_{E}$ symmetry is accomplished by the addition to the Lagrangian of three extra fermion species, denoted here by $\mathcal{E}_{R}, \mathcal{E}_{L}$, and $\mathcal{N}_{R}$. Their quantum numbers are shown in table 1, together with those for the SM fields.

In addition, for all fermion bi-linears invariant under the SM gauge symmetry but not under the flavour symmetry, a scalar is introduced to restore flavour invariance. Only two such scalar flavon fields are needed, denoted by $\mathcal{Y}_{E}$ and $\mathcal{Y}_{N}$ in table 1 , belonging respectively to the bi-fundamental representation of $\mathrm{SU}(3)_{\ell} \times \mathrm{SU}(3)_{E}$ and to the conjugatesymmetric representation of $\mathrm{SU}(3)_{\ell}$. The vevs of these fields are related to the Yukawa matrices but should not be directly identified with them, as functions of the flavon fields may have the same transformation properties under flavour than $\mathcal{Y}_{E, N}$, and they also allow to build flavour invariant Lagrangian terms; ${ }^{1}$ this is a property essential to the phenomenological success of the construction. Finally, other scalars charged under the SM gauge group are not considered since they would not respect the condition of minimality of the spectrum, in addition to potentially disrupting the electroweak symmetry breaking (EWSB) mechanism.

Within this framework, the most general renormalizable Lagrangian with $\mathrm{SU}(3)_{\ell} \times$ $\mathrm{SU}(3)_{E}$ gauge symmetry therefore reads:

$$
\begin{aligned}
\mathscr{L}= & i \sum_{\psi} \bar{\psi} \not D \psi-\frac{1}{2} \sum_{I} \operatorname{Tr}\left(F_{\mu \nu}^{I} F_{I}^{\mu \nu}\right)+\sum_{B} \operatorname{Tr}\left(D_{\mu} \mathcal{Y}_{B} D^{\mu} \mathcal{Y}_{B}^{\dagger}\right)+D_{\mu} H^{\dagger} D^{\mu} H \\
& +\mathscr{L}_{Y}-V\left(H, \mathcal{Y}_{E}, \mathcal{Y}_{N}\right)
\end{aligned}
$$

where $\psi$ runs over all lepton species in table $1, I=\ell, E$ and $B$ identifies flavon indices $B=E, N$. The gauge bosons of $\mathrm{SU}(3)_{\ell}$ and $\mathrm{SU}(3)_{E}$ will be encoded in traceless hermitian

\footnotetext{
${ }^{1}$ For instance $\left(\mathcal{Y}_{E}^{-1}\right)^{\dagger}$ and $\mathcal{Y}_{E}$ belong to the same flavour representation.
} 
matrices in flavour space, $A_{\mu}^{\ell}$ with $A_{\mu, \alpha \beta}^{\ell}=\left(A_{\mu, \beta \alpha}^{\ell}\right)^{*}, \Sigma_{\alpha} A_{\mu, \alpha \alpha}^{\ell}=0$, and $A_{\mu}^{E}$ with $A_{\mu, \alpha \beta}^{E}=$ $\left(A_{\mu, \beta \alpha}^{E}\right)^{*}, \Sigma_{\alpha} A_{\mu, \alpha \alpha}^{E}=0$, which can be alternatively decomposed in terms of generators

$$
A_{\mu}^{\ell} \equiv \sum_{a=1}^{8} A_{\mu}^{\ell, a} T^{a}, \quad A_{\mu}^{E} \equiv \sum_{a=1}^{8} A_{\mu}^{E, a} T^{a},
$$

where $T^{a}$ are the flavour group generators, with $\operatorname{Tr}\left(T^{a} T^{b}\right)=\delta^{a b} / 2$ and $T^{a} \equiv \lambda_{\mathrm{SU}(3)}^{a} / 2$, and $\lambda_{\mathrm{SU}(3)}^{a}$ denote the Gell-Mann matrices. The gauge couplings of $A_{\mu}^{\ell}$ and $A_{\mu}^{E}$ will be denoted by $g_{\ell}$ and $g_{E}$, respectively. In eq. (2.2) the field strengths include those for the SM fields and flavour gauge bosons, as do the covariant derivatives, e.g.

$$
D_{\mu} \ell_{L}=\left(\partial_{\mu}-i \frac{g^{\prime}}{2} B_{\mu}+i \frac{g}{2} \sigma_{I} W_{\mu}^{I}+i g_{\ell} A_{\mu}^{\ell}\right) \ell_{L},
$$

while

$$
\begin{aligned}
& D_{\mu} \mathcal{Y}_{E}=\partial_{\mu} \mathcal{Y}_{E}+i g_{E} A_{\mu}^{E} \mathcal{Y}_{E}-i g_{\ell} \mathcal{Y}_{E} A_{\mu}^{\ell}, \\
& D_{\mu} \mathcal{Y}_{N}=\partial_{\mu} \mathcal{Y}_{N}-i g_{\ell}\left(A_{\mu}^{\ell}\right)^{T} \mathcal{Y}_{N}-i g_{\ell} \mathcal{Y}_{N} A_{\mu}^{\ell} .
\end{aligned}
$$

The Yukawa and mass terms can be written as follows:

$$
\begin{aligned}
\mathscr{L}_{Y}= & \lambda_{E} \bar{\ell}_{L} H \mathcal{E}_{R}+\mu_{E} \overline{\mathcal{E}}_{L} e_{R}+\lambda_{\mathcal{E}} \overline{\mathcal{E}}_{L} \mathcal{Y}_{E} \mathcal{E}_{R}+\text { h.c. } \\
& +\lambda_{\nu} \bar{\ell}_{L} \tilde{H} \mathcal{N}_{R}+\frac{\lambda_{N}}{2} \overline{\mathcal{N}_{R}{ }^{c}} \mathcal{Y}_{N} \mathcal{N}_{R}+\text { h.c. }
\end{aligned}
$$

where $\ell_{L}, e_{R}, \mathcal{E}_{L}, \mathcal{E}_{R}$ and $\mathcal{N}_{R}$ are vectors in flavour space. $\lambda_{E}, \lambda_{\mathcal{E}}, \lambda_{\nu}, \lambda_{N}$ and $\mu_{E}$ are each a single complex parameter, since these couplings must be proportional to the identity to preserve flavour invariance; moreover they can be made real and positive via chiral fermion transformations. In contrast, $\mathcal{Y}_{E}$ and $\mathcal{Y}_{N}$ are matrices in flavour space and their nontrivial background values are the only sources of flavour (including $\mathrm{CP}$ violation). Notice that $\mu_{E}$ is not the mass of any of the particles in the spectrum, but simply a mass parameter of the Lagrangian. The vev of $\mathcal{Y}_{N}$ is simultaneously the LN scale and the flavour scale; in the limit $\mathcal{Y}_{N}=0$ in which only (diagonal) Dirac mass terms remain, the Lagrangian would acquire a $\mathrm{U}(1)_{e} \times \mathrm{U}(1)_{\mu} \times \mathrm{U}(1)_{\tau}$ symmetry which prevents the appearance of leptonic mixing angles, a setup phenomenologically not viable. For this reason the introduction of $\mathcal{Y}_{N}$ is necessary and therefore Majorana neutrino masses follow as a natural consequence of gauging flavour in the lepton sector, even when taking as starting point only the SM gauge symmetry.

The above Lagrangian has two accidental U(1) symmetries which are anomaly free under the flavour gauge group. The first is an extension of LN symmetry, under which all fermions transform with the same charge while $\mathcal{Y}_{N}$ transforms with minus twice that charge. The second accidental symmetry is the abelian $\mathrm{U}(1)_{E}$ acting on right-handed charged leptons, that completes $\mathrm{SU}(3)_{E}$ to a unitary group, and under which $e_{R}, \mathcal{E}_{L}$ and $\mathcal{Y}_{E}$ transform non-trivially. Both U(1)'s would be spontaneously broken by the scalar vevs. However, in all generality, the scalar potential contains terms such as $\operatorname{det}\left(\mathcal{Y}_{E}\right)$ and $\operatorname{det}\left(\mathcal{Y}_{N}\right)$ [11], that break explicitly these U(1)'s and prevent the appearance of phenomenologically dangerous Goldstone bosons. 
In order to yield masses for all fermions, $\mathscr{L}_{Y}$ in eq. (2.6) must undergo both EWSB and flavour symmetry breaking, so that in the unitary gauge

$$
\begin{aligned}
H & \equiv(v+h) / \sqrt{2} \\
\mathcal{Y}_{E} & \equiv\left\langle\mathcal{Y}_{E}\right\rangle+\phi_{E} / \sqrt{2} \\
\mathcal{Y}_{N} & \equiv\left\langle\mathcal{Y}_{N}\right\rangle+\phi_{N} / \sqrt{2}
\end{aligned}
$$

where $h$ denotes the physical Higgs particle and $\phi_{E}$ and $\phi_{N}$ the physical scalar excitations over the flavon vevs $\left\langle\mathcal{Y}_{E}\right\rangle \neq 0,\left\langle\mathcal{Y}_{N}\right\rangle \neq 0$ (for simplicity, the Yukawa flavons and their vevs will be denoted with the same symbols in the next sections). The ensuing spectrum contains 6 Dirac electro-magnetically charged fermions and 6 Majorana neutral fermions. There are no extra scalars charged under the SM gauge group and EWSB proceeds thus as usual. The dynamics of flavour breaking is encoded in the scalar potential, which has been studied in refs. $[11,13,14]$. The study of the potential is involved due to the complex flavour structure that it aims to explain, but some general results and approximately conserved symmetries where found in refs. $[11,13,14]$. In particular, a connection between degenerate spectra with large angles and maximal Majorana phases was found for the neutrino sector.

\subsection{Spectrum}

Fermions. The Lagrangian in eq. (2.6) results in leptonic mass matrices for charged and neutral leptons of the form

$$
\left(\begin{array}{cc}
0 & \lambda_{E} v / \sqrt{2} \\
\mu_{E} & \lambda_{\mathcal{E}} \mathcal{Y}_{E}
\end{array}\right)+\text { h.c., } \quad \frac{1}{2}\left(\begin{array}{cc}
0 & \lambda_{\nu} v / \sqrt{2} \\
\lambda_{\nu} v / \sqrt{2} & \lambda_{N} \mathcal{Y}_{N}
\end{array}\right)+\text { h.c. },
$$

respectively, which suggest immediately a Seesaw-like pattern for both sectors. No additional fermions beyond those in the SM have been detected at experiments and this fact sets strong bounds on the mass of the mirror fermions $\mathcal{E}$ and $\mathcal{N}$ introduced for the sake of flavour anomaly cancellation. This indicates that the mass term for the extra charged leptons, $\lambda_{\mathcal{E}} \mathcal{Y}_{E}$, should be larger than the other scales of the theory: $\mathcal{Y}_{E} \gg \mu_{E}, v,-$ assuming all dimensionless parameters to be $\mathcal{O}(1)$. This is analogous to the condition for neutrinos $\mathcal{Y}_{N} \gg v$ in the canonical type I Seesaw model on the right-hand side of eq. (2.8), which leads to a mass scale of order $\sim 10^{12} \mathrm{GeV}$ for the extra neutral fermions. With these approximations, the Lagrangian in eq. (2.6) yields a Dirac mass for the heavy charged leptons $\mathcal{E}$ and a Majorana mass for the right-handed singlets,

$$
\mathcal{M}_{\mathcal{E}}=\lambda_{\mathcal{E}} \mathcal{Y}_{E}\left(1+\mathcal{O}\left(\frac{v^{2}}{\mathcal{Y}_{E}^{2}}, \frac{\mu_{E}^{2}}{\mathcal{Y}_{E}^{2}}\right)\right), \quad \mathcal{M}_{N}=\lambda_{N} \mathcal{Y}_{N}\left(1+\mathcal{O}\left(\frac{v^{2}}{\mathcal{Y}_{N}^{2}}\right)\right)
$$

where $\mathcal{M}_{\mathcal{E}}$ and $\mathcal{M}_{N}$ denote the heavy lepton mass matrices while the mass matrices for the light states obey (see eq. (1.4))

$$
\begin{gathered}
Y_{E}=\frac{m_{\ell}}{v / \sqrt{2}}=\frac{\lambda_{E}}{\lambda_{\mathcal{E}}}\left(\frac{\mu_{E}}{\mathcal{Y}_{E}}\right)\left(1+\mathcal{O}\left(\frac{v^{2}}{\mathcal{Y}_{E}^{2}}, \frac{\mu_{E}^{2}}{\mathcal{Y}_{E}^{2}}\right)\right), \\
\frac{C_{\nu}}{\Lambda_{\mathrm{LN}}}=\frac{m_{\nu}}{v^{2} / 2}=\lambda_{\nu}\left(\frac{1}{\lambda_{N} \mathcal{Y}_{N}}\right) \lambda_{\nu}\left(1+\mathcal{O}\left(\frac{v^{2}}{\mathcal{Y}_{N}^{2}}\right)\right),
\end{gathered}
$$



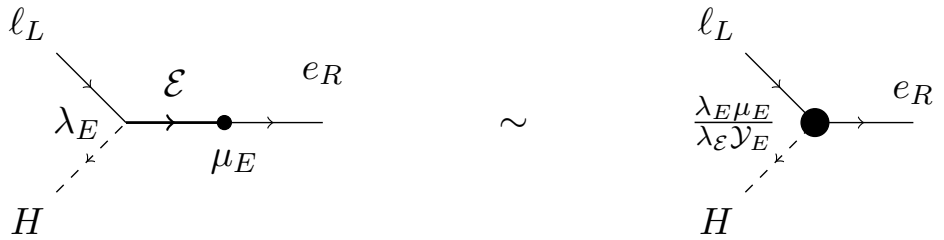

Figure 1. Diagrammatic representation of the generation of SM charged lepton Yukawa couplings (right figure) induced by the exchange of heavy mirror charged leptons (left figure).

illustrating that the mirror fermions are proportional to the flavon vevs while SM fermion masses are inversely proportional to them. It follows that

$$
m_{\ell} \mathcal{M}_{\mathcal{E}} \approx \lambda_{E} \mu_{E} v / \sqrt{2}, \quad m_{\nu} \mathcal{M}_{N} \approx \lambda_{\nu}^{2} v^{2} / 2 .
$$

The masses of the SM leptons are thus shown to be related to those of the heaviest extra leptons by an inverse proportionality law: a Seesaw mechanism is present both for charged and neutral leptons, similar to the case of quarks in ref. [15].

All flavour structure being encoded in $\mathcal{Y}_{E}$ and $\mathcal{Y}_{N}$, their eigenvalues determine the hierarchy of lepton masses up to common factors:

$$
\begin{aligned}
& M_{\mathcal{E}} \equiv\left(M_{\hat{e}}, M_{\hat{\mu}}, M_{\hat{\tau}}\right) \simeq \lambda_{E} \mu_{E}\left(3.5 \cdot 10^{5}, 1.7 \cdot 10^{3}, 10^{2}\right), \\
& M_{N} \equiv\left(M_{1}, M_{2}, M_{3}\right)<\left|\lambda_{\nu}\right|^{2} \frac{v}{\sqrt{2}}\left(\infty, 2 \cdot 10^{13}, 3.5 \cdot 10^{12}\right),
\end{aligned}
$$

where $M_{\mathcal{E}}\left(M_{N}\right)$ denotes the diagonal matrix of eigenvalues of the $\mathcal{M}_{\mathcal{E}}\left(\mathcal{M}_{N}\right)$ matrix and the hat refers to the individual charged mirror fermions masses. ${ }^{2}$

The expressions for the SM lepton masses can be also derived diagrammatically by integrating out the heavy states as shown in figure 1 for charged leptons. It illustrates that all light flavour structure stems from the mass matrix of mirror leptons given by $\mathcal{Y}_{E}$, as the equivalent of the usual Yukawa couplings, $\lambda_{E}$ and $\lambda_{\mathcal{E}}$, as well as $\mu_{E}$, are overall constants. This resembles the MFV scenario of ref. [30] that, however, leads to different phenomenology, see section 4 .

From now on, we will work on a basis in which the charged lepton mass matrix $\mathcal{Y}_{E}$ is diagonal, and thus $\mathcal{M}_{\mathcal{E}}=M_{\mathcal{E}}$. For later use, it is convenient to explicitly invert the relations in eq. (2.10) to extract the expressions for the flavon vevs,

$$
\mathcal{Y}_{E}=\frac{\lambda_{E} \mu_{E}}{\sqrt{2} \lambda_{\mathcal{E}}} \operatorname{diag}\left(\frac{v}{m_{e}}, \frac{v}{m_{\mu}}, \frac{v}{m_{\tau}}\right), \quad \mathcal{Y}_{N}=\frac{\lambda_{\nu}^{2} v}{2 \lambda_{N}} U^{*} \operatorname{diag}\left(\frac{v}{m_{\nu_{1}}}, \frac{v}{m_{\nu_{2}}}, \frac{v}{m_{\nu_{3}}}\right) U^{\dagger},
$$

where $U$ is the PMNS leptonic mixing matrix. Notice that the choice of basis is allowed by the flavour symmetry without loss of generality. The flavon vevs are thus determined by low energy flavour data up to an overall constant.

\footnotetext{
${ }^{2}$ The unknown absolute neutrino mass scale translates in an inequality in contrast with the case of charged leptons, and a bound on $M_{1}$ cannot be derived since one neutrino could be massless.
} 


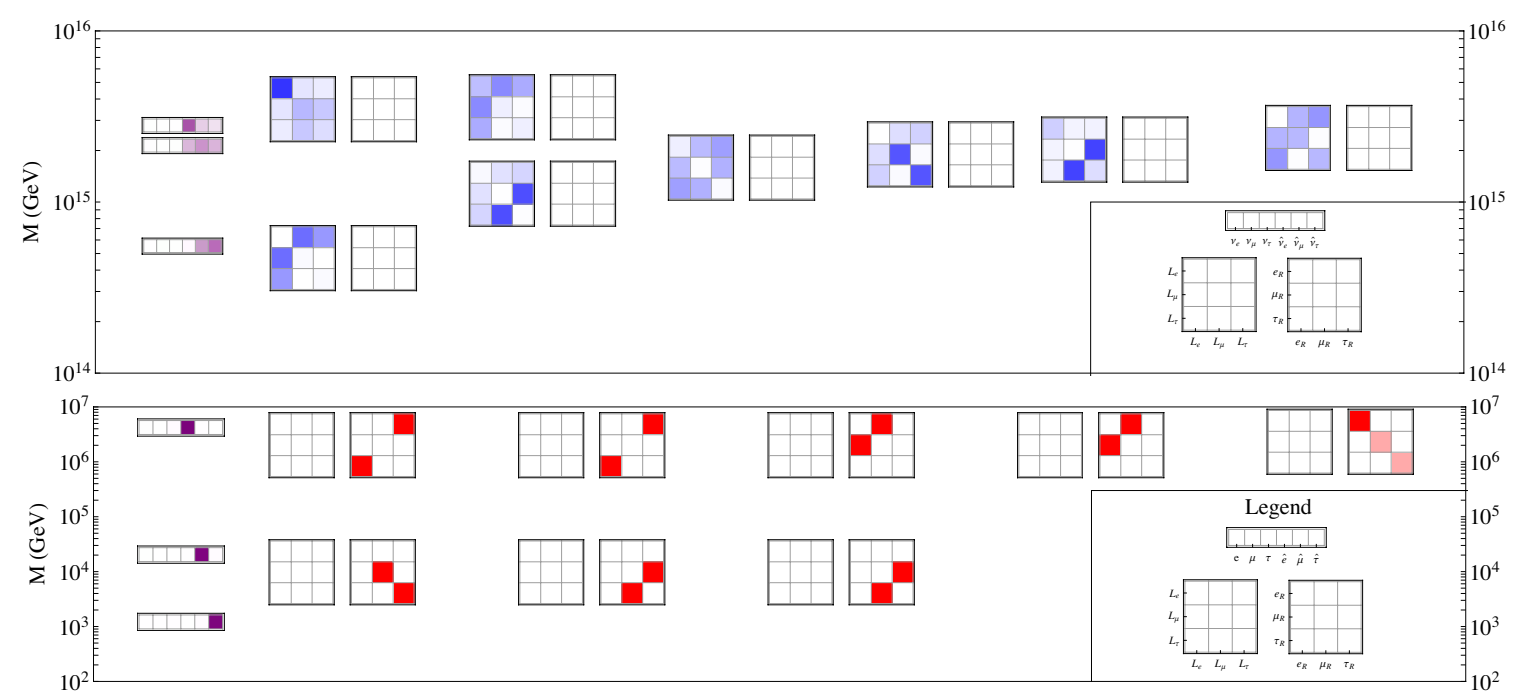

Figure 2. Gauge and fermion heavy spectrum for the gauged SM lepton flavour. Boxes composed out of $3 \times 3$ squares depict the gauge boson mass eigenstates and rows of squares depict mirror fermions. For the first, the squares are ordered according to the $e, \mu$ and $\tau$ flavour, from left to right and from top to bottom. The boxes in the upper panel correspond dominantly to the $\mathrm{SU}(3)_{\ell}$ symmetry, with the gauge bosons shown in blue, while the lower panel shows in red the $\mathrm{SU}(3)_{E}$ gauge bosons. In both cases the intensity of the coloured cells represents the strength of the coupling between the gauge boson and each lepton bilinear. As for the fermions, the intensity of the cells represents, from left to right, the component of $e, \mu, \tau, \hat{e}, \hat{\mu}$ and $\hat{\tau}$ for the lower panel, and of $\nu_{e}$, $\nu_{\mu}, \nu_{\tau}, \nu_{\hat{e}}, \nu_{\hat{\mu}}$, and $\nu_{\hat{\tau}}$ in the upper panel. Normal ordering was assumed for neutrinos and the parameter values used are $\theta_{23}=45^{\circ}, \theta_{12}=33^{\circ}, \theta_{13}=8.8^{\circ}$, Dirac CP phase $\delta=2 \pi / 3$, Majorana phases $\alpha_{1}=\alpha_{2}=0$, lightest neutrino mass $m_{\nu_{1}}=10^{-11} \mathrm{GeV}$; all flavour gauge coupling constants and all $\lambda$ 's are set to 0.1 , with $\mu_{E}=15 \mathrm{GeV}$.

The spectrum of mirror fermions is illustrated as horizontal lines on the left-hand side of figure 2 for natural values of the parameters. As anticipated, due to the inverse dependence of mirror lepton masses with respect to their light counterparts the lightest exotic fermion is the $\tau$ mirror lepton. The $\mu$ mirror lepton appears next, a factor $\sim m_{\tau} / m_{\mu}$ higher. The mirror $e$ appears yet a factor $m_{\mu} / m_{e}$ above. Much higher in mass by a factor $\sim m_{e} / m_{\nu}$, the mirror neutrinos 3, 2 and 1 appear (in this illustration normal ordering was assumed for the light neutrinos).

Flavoured gauge bosons. Flavour symmetry breaking produces masses for the sixteen flavour gauge bosons encoded in $A_{\mu}^{\ell}$ and $A_{\mu}^{E}$. The relevant part of the Lagrangian, including only terms at most quadratic in the gauge fields, is given by

$$
\begin{aligned}
& \sum_{I=\ell, E} \operatorname{Tr}\left(A_{\mu}^{I} \partial^{2} A^{I, \mu}\right)+\operatorname{Tr}\left\{\left(g_{E} A_{\mu}^{E} \mathcal{Y}_{E}-g_{\ell} \mathcal{Y}_{E} A_{\mu}^{\ell}\right)\left(g_{E} \mathcal{Y}_{E}^{\dagger} A^{E, \mu}-g_{\ell} A^{\ell, \mu} \mathcal{Y}_{E}^{\dagger}\right)\right\} \\
& +g_{\ell}^{2} \operatorname{Tr}\left\{\left(A_{\mu}^{\ell *} \mathcal{Y}_{N}+\mathcal{Y}_{N} A_{\mu}^{\ell}\right)\left(\mathcal{Y}_{N}^{\dagger}\left(A^{\ell, \mu}\right)^{T}+A^{\ell, \mu} \mathcal{Y}_{N}^{\dagger}\right)\right\}-\sum_{I} g_{I} \operatorname{Tr}\left(A_{\mu}^{I} J_{A^{I}}^{\mu}\right),
\end{aligned}
$$


where the currents are hermitian matrices in flavour space:

$$
\begin{aligned}
& {\left[J_{A^{\ell}}^{\mu}\right]_{i j}=\bar{\ell}_{L}^{j} \gamma^{\mu} \ell_{L}^{i}+\overline{\mathcal{E}}_{R}^{j} \gamma^{\mu} \mathcal{E}_{R}^{i}+\overline{\mathcal{N}_{R}^{j}} \gamma^{\mu} \mathcal{N}_{R}^{i},} \\
& {\left[J_{A^{E}}^{\mu}\right]_{i j}=\bar{e}_{R}^{j} \gamma^{\mu} e_{R}^{i}+\overline{\mathcal{E}}_{L}^{j} \gamma^{\mu} \mathcal{E}_{L}^{i},}
\end{aligned}
$$

where $i, j$ are flavour indices. The linear equations of motion (EOMs) in matrix form stemming from eq. (2.15) reads

$$
\begin{aligned}
& \partial^{2} A_{\mu}^{\ell}-g_{E} g_{\ell} \mathcal{Y}_{E}^{\dagger} A_{\mu}^{E} \mathcal{Y}_{E}+\frac{g_{\ell}^{2}}{2}\left\{\mathcal{Y}_{E}^{\dagger} \mathcal{Y}_{E}\right.\left.+\mathcal{Y}_{N}^{\dagger} \mathcal{Y}_{N}+\mathcal{Y}_{N}^{*} \mathcal{Y}_{N}^{T}, A_{\mu}^{\ell}\right\} \\
&+2 g_{\ell}^{2} \mathcal{Y}_{N}^{\dagger} A_{\mu}^{\ell *} \mathcal{Y}_{N}-\frac{g_{\ell}}{2} J_{\mu}^{A^{\ell}}=\frac{1}{n_{g}} \operatorname{Tr} \text { (L.H.S.) } \mathbb{1}, \\
& \partial^{2} A_{\mu}^{E}-g_{E} g_{\ell} \mathcal{Y}_{E} A_{\mu}^{\ell} \mathcal{Y}_{E}^{\dagger}+\frac{g_{E}^{2}}{2}\left\{\mathcal{Y}_{E} \mathcal{Y}_{E}^{\dagger}, A_{\mu}^{E}\right\}-\frac{g_{E}}{2} J_{\mu}^{A^{E}}=\frac{1}{n_{g}} \operatorname{Tr} \text { (L.H.S.) } \mathbb{1},
\end{aligned}
$$

where $\{\ldots, \ldots\}$ denotes the anti-commutator, $n_{g}=3$ and L.H.S. stands for left hand side. ${ }^{3}$ These equations can be alternatively written as an inhomogeneous linear system for the sixteen gauge fields when the latter are described as an array of sixteen $\chi_{\mu}^{a}$ fields,

$$
\chi_{\mu} \equiv\left(A_{\mu}^{\ell, 1}, \ldots, A_{\mu}^{\ell, 8}, A_{\mu}^{E, 1}, \ldots, A_{\mu}^{E, 8}\right),
$$

which allows to rewrite the Lagrangian in eq. (2.15) as

$$
\mathscr{L}_{\text {gauge }}=-\frac{1}{2} \sum_{I=\ell, E} \operatorname{Tr}\left(F_{\mu \nu}^{I} F_{I}^{\mu \nu}\right)+\frac{1}{2} \sum_{a, b=1}^{16} \chi_{\mu}^{a}\left(M_{A}^{2}\right)_{a b} \chi^{b, \mu}-\sum_{I=\ell, E} g_{I} \operatorname{Tr}\left(A_{\mu}^{I} J_{A_{I}}^{\mu}\right),
$$

where the mass matrix $M_{A}$ can be expressed as

$$
M_{A}^{2}=\left(\begin{array}{cc}
M_{\ell \ell}^{2} & M_{\ell E}^{2} \\
M_{E \ell}^{2} & M_{E E}^{2}
\end{array}\right)
$$

with

$$
\begin{aligned}
\left(M_{\ell \ell}^{2}\right)_{i j}= & g_{\ell}^{2}\left\{\operatorname{Tr}\left(\mathcal{Y}_{E}\left\{T_{i}, T_{j}\right\} \mathcal{Y}_{E}^{\dagger}\right)+\operatorname{Tr}\left(\mathcal{Y}_{N}\left\{T_{i}, T_{j}\right\} \mathcal{Y}_{N}^{\dagger}\right)\right. \\
& \left.+\operatorname{Tr}\left(\mathcal{Y}_{N}^{\dagger}\left\{T_{i}^{T}, T_{j}^{T}\right\} \mathcal{Y}_{N}\right)+2 \operatorname{Tr}\left(\mathcal{Y}_{N}^{\dagger} T_{i}^{T} \mathcal{Y}_{N} T_{j}+\mathcal{Y}_{N}^{\dagger} T_{j}^{T} \mathcal{Y}_{N} T_{i}\right)\right\}, \\
\left(M_{\ell E}^{2}\right)_{i j}=\left(M_{E \ell}^{2}\right)_{j i}=-2 g_{\ell} g_{E} \operatorname{Tr}\left(T_{i} \mathcal{Y}_{E}^{\dagger} T_{j} \mathcal{Y}_{E}\right), & \\
\left(M_{E E}^{2}\right)_{i j}= & g_{E}^{2} \operatorname{Tr}\left(\mathcal{Y}_{E}^{\dagger}\left\{T_{i}, T_{j}\right\} \mathcal{Y}_{E}\right),
\end{aligned}
$$

where $i, j=\{1, \ldots, 8\}$, and the linear EOM can be now written in the customary form,

$$
\left(\partial^{2}+M_{A}^{2}\right) \chi^{\mu}=J_{A}^{\mu}, \quad \text { where } \quad J_{\mu}^{A} \equiv\left(J_{A^{\ell}}^{\mu, 1}, \ldots, J_{A^{\ell}}^{\mu, 8}, J_{A^{E}}^{\mu, 1}, \ldots, J_{A^{E}}^{\mu, 8}\right) .
$$

Eq. (2.22) shows that gauge boson masses are proportional to the scalar fields $\mathcal{Y}_{E}$ and $\mathcal{Y}_{N}$ whose structure is in turn given by, and inversely proportional to, light fermion masses and mixings, see eq. (2.14). The spectrum of sixteen mass states is thus determined up to two overall constants, that can be identified with the products $g_{E}\left\|\mathcal{Y}_{E}\right\|, g_{\ell}\left\|\mathcal{Y}_{N}\right\| .{ }^{4}$ The

\footnotetext{
${ }^{3}$ Eq. (2.18) displays explicitly the covariant properties of the gauge bosons and the trace removes the singlet component of each term, leaving only the adjoint combination to which the gauge bosons belong.

${ }^{4}$ The modulus of a matrix $B$ is defined as $\|B\|^{2} \equiv \operatorname{Tr}\left(B^{\dagger} B\right)$, implying that $\left\|\mathcal{Y}_{E}\right\|$ and $\left\|\mathcal{Y}_{N}\right\|$ are flavour invariant constructions.
} 
hierarchy $\mathcal{Y}_{N} \gg \mathcal{Y}_{E}$ that followed from assuming order one dimensionless coefficients and $\mu_{E}$ around the EW scale, implies that:

- The heaviest gauge bosons to good approximation are those of the $\mathrm{SU}(3)_{\ell}$ group, $A_{\mu}^{\ell}$, while the lightest gauge bosons will be those corresponding to the $\mathrm{SU}(3)_{E}$ group, $A_{\mu}^{E}$.

- In this regime the mixing between $A_{\mu}^{E}$ and $A_{\mu}^{\ell}$ is small. We will refer to $A_{\mu}^{E}\left(A_{\mu}^{\ell}\right)$ as the lightest (heaviest) states.

The spectrum of flavour gauge bosons is shown in figure 2 next to that for mirror fermions, for natural values of the parameters. Boxes represent flavour gauge bosons and the colored entries in a given box indicate the lepton flavours to which that gauge boson couples. The blue-colored boxes in the upper panel correspond to the $A_{\mu}^{\ell}$ gauge bosons, while the red-colored boxes correspond to the $A_{\mu}^{E}$ gauge bosons; as expected the former are heavier by a factor $\sim m_{e} / m_{\nu}$ due to the inverse dependence of their masses with the light neutrino mass.

Lightest gauge bosons. The $A_{\mu}^{E}$ fields will thus dominate the phenomenology mediated by flavour gauge bosons. Because their mass matrix is to a good approximation proportional to the charged lepton flavon vev $\mathcal{Y}_{E}$, while the charged lepton mass matrix is instead inversely proportional to it, the hierarchies in charged lepton masses translate into hierarchies in the gauge boson spectrum: the lightest $A_{\mu}^{E}$ gauge bosons will be those mediating transitions which involve the heaviest right-handed charged leptons and in particular the $\tau_{R}$ lepton. In fact, because of the zero trace of the generators, at least two different leptons must participate in any coupling, and the overall conclusion is that the lightest flavour gauge bosons will produce deviations in both $\mu_{R}$ and $\tau_{R}$ sectors.

Technically, the $A_{\mu}^{E}$ mass eigenstates are largely aligned with the $\mathrm{SU}(3)$ generators except for the diagonal components given by

$$
\hat{T}_{3} \equiv\left(\sqrt{3} T_{8}-T_{3}\right) / 2=\frac{1}{2}\left(\begin{array}{ccc}
0 & 0 & 0 \\
0 & 1 & 0 \\
0 & 0 & -1
\end{array}\right), \quad \hat{T}_{8} \equiv\left(\sqrt{3} T_{3}+T_{8}\right) / 2=\frac{1}{2 \sqrt{3}}\left(\begin{array}{ccc}
2 & 0 & 0 \\
0 & -1 & 0 \\
0 & 0 & -1
\end{array}\right) .
$$

It follows from eq. (2.22) that their respective masses are given by

$$
M_{A_{\mu}^{E, a}}^{2} \simeq 2 g_{E}^{2} \frac{\left\|\hat{T}_{a} m_{\ell}^{-1}\right\|^{2}}{\left\|\mathcal{Y}_{E}^{-1}\right\|^{2}} \sum_{\alpha=e, \mu, \tau} m_{\alpha}^{2}
$$

where $\hat{T}^{a}=T^{a}$ for all $a \neq 3,8, m_{\ell}$ is the mass matrix of the charged leptons and greek indices stand from now on for charged lepton flavours. The fact that the size of $\mathcal{Y}_{E}\left(\mathcal{Y}_{E}^{-1}\right)$ is dominated by the electron (tau) mass,

$$
\left\|\mathcal{Y}_{E}\right\|^{2}=\frac{\lambda_{E}^{2} \mu_{E}^{2}}{2 \lambda_{\mathcal{E}}^{2}} \frac{v^{2}}{m_{e}^{2}}\left(1+\frac{m_{e}^{2}}{m_{\mu}^{2}}+\frac{m_{e}^{2}}{m_{\tau}^{2}}\right), \quad\left\|\mathcal{Y}_{E}^{-1}\right\|^{2}=\frac{2 \lambda_{\mathcal{E}}^{2}}{\lambda_{E}^{2} \mu_{E}^{2}} \frac{m_{\tau}^{2}}{v^{2}}\left(1+\frac{m_{\mu}^{2}}{m_{\tau}^{2}}+\frac{m_{e}^{2}}{m_{\tau}^{2}}\right)
$$


makes all gauge bosons with a right-handed electron entry a factor $m_{\mu} / m_{e}$ heavier than the rest. Indeed, because $m_{e} \ll m_{\mu}, m_{\tau}$, this can be seen as an approximate $\mathrm{SU}(2)$ symmetry in the $\mu-\tau$ sector when $\mathcal{Y}_{E}$ is taken to be $\operatorname{diag}\left(1 / y_{e}, 0,0\right)$, which is the reason why the diagonal generators $\hat{T}_{8}, \hat{T}_{3}$ are better suited to describe mass states than $T_{8}, T_{3}$. Moreover, under the $\mathrm{U}(1)_{e} \times \mathrm{U}(1)_{\mu} \times \mathrm{U}(1)_{\tau}$ approximate symmetry present for $\mathcal{Y}_{N} \gg \mathcal{Y}_{E}$, the off-diagonal gauge bosons transform as $A_{\alpha \beta}^{E} \rightarrow e^{i \theta_{\alpha}-i \theta_{\beta}} A_{\alpha \beta}^{E}$, which requires that both components of each off-diagonal entry have the same mass (so as to combine into a complex gauge boson): this approximate symmetry will suppress charged lepton flavour violation.

In summary, the three $A_{\mu}^{E}$ gauge bosons corresponding to the approximate $\mathrm{SU}(2)$ symmetry in the $\mu-\tau$ sector are found to be the lightest (first layer of the lower panel in figure 2); a factor $m_{\mu} / m_{e}$ higher the remaining five $\mathrm{SU}(3)_{E}$ gauge bosons appear (second layer in that figure). In turn, the leading phenomenological signals consists of flavourconserving leptonic observables and, furthermore, low energy processes mediated by $A_{\mu}^{E}$ for the lighter leptons are suppressed by heavier mass scales, providing a flavour protection mechanism, as previously described for quarks in ref. [15].

As for the relative mass of mirror fermions versus flavour gauge bosons, the lightest particle turns out to be the mirror tau lepton $\hat{\tau}$, see figure 2. Indeed, the lightest gauge boson mass $\sim\left(g_{E} /\left\|\mathcal{Y}_{E}^{-1}\right\|\right)\left(m_{\tau} / m_{\mu}\right)$ is a factor $\sim m_{\tau} / m_{\mu}$ larger than the lightest mirror fermion mass $\sim \lambda_{\mathcal{E}} /\left\|\mathcal{Y}_{E}^{-1}\right\|$, due to the tracelessness of the generators implying a nonvanishing $\mu \mu$ or $\mu \tau$ entry in the three lightest gauge boson interactions. In contrast, were the full $\mathrm{U}(3)_{E}$ group gauged an associated lighter $\left(A_{\mu}^{E}\right)_{\tau \tau}$ gauge boson would appear in the spectrum.

Scalars. Flavour symmetry breaking gives rise to $18\left(\mathcal{Y}_{E}\right)+12\left(\mathcal{Y}_{N}\right)-16\left(\mathrm{SU}(3)^{2}\right)=14$ physical scalar bosons, corresponding to fluctuations around the 6 mixing parameters, 6 masses and $\mathrm{U}(1)_{\ell}$ and $\mathrm{U}(1)_{E}$ phases. This part of the spectrum will in general contribute to the same observables than flavour gauge bosons, although without disrupting the flavour structure [15]. The detailed scalar mass spectrum depends however on the scalar potential parameters, as opposed to the gauge bosons and fermions, and it will not be discussed further in this work.

\subsection{Interactions}

The distinction between fermionic mass and interaction eigenstates will be relevant: therefore, for the rest of this section flavour eigenstates will be denoted with a prime ${ }^{5}$ and described by

$$
\begin{aligned}
\left(\begin{array}{c}
e_{L}^{\prime} \\
\mathcal{E}_{L}^{\prime}
\end{array}\right) & =\left(\begin{array}{cc}
c_{\Theta} & s_{\Theta} \\
-s_{\Theta^{\dagger}} & c_{\Theta^{\dagger}}
\end{array}\right)\left(\begin{array}{c}
e_{L} \\
\mathcal{E}_{L}
\end{array}\right), \quad\left(\begin{array}{c}
e_{R}^{\prime} \\
\mathcal{E}_{R}^{\prime}
\end{array}\right)=\left(\begin{array}{cc}
c_{\Theta_{R}^{\dagger}} & -s_{\Theta_{R}^{\dagger}} \\
-s_{\Theta_{R}} & -c_{\Theta_{R}}
\end{array}\right)\left(\begin{array}{c}
e_{R} \\
\mathcal{E}_{R}
\end{array}\right), \\
\left(\begin{array}{c}
\nu_{L}^{c \prime} \\
\mathcal{N}_{R}^{\prime}
\end{array}\right) & =\left(\begin{array}{cc}
c_{\Theta_{\nu}^{\dagger}} & i s_{\Theta_{\nu}^{\dagger}} \\
-s_{\Theta_{\nu}} & i c_{\Theta_{\nu}}
\end{array}\right)\left(\begin{array}{c}
\nu_{L}^{c} \\
\mathcal{N}_{R}
\end{array}\right),
\end{aligned}
$$

where unprimed fields are here mass eigenstates and the mixing angles are encoded in $3 \times 3$ matrices in flavour space $\Theta, c_{\Theta}=(-1)^{n} /(2 n) !\left(\Theta \Theta^{\dagger}\right)^{n}, s_{\Theta}=(-1)^{n} \Theta /(2 n+1) !\left(\Theta^{\dagger} \Theta\right)^{n}[36]$.

\footnotetext{
${ }^{5}$ For instance, all fermions in table 1 will be considered as primed fields for the sake of this section.
} 
These unitary rotations diagonalize the mass terms stemming from eq. (2.6) (see also eqs. (2.9) and eqs. (2.10)):

$$
\begin{array}{r}
-\left(\begin{array}{cc}
c_{\Theta} & -s_{\Theta} \\
s_{\Theta^{\dagger}} & c_{\Theta^{\dagger}}
\end{array}\right)\left(\begin{array}{cc}
0 & \lambda_{E} v / \sqrt{2} \\
\mu_{E} & \lambda_{\mathcal{E}} \mathcal{Y}_{E}
\end{array}\right)\left(\begin{array}{cc}
c_{\Theta_{R}^{\dagger}} & -s_{\Theta_{R}^{\dagger}}^{\dagger} \\
-s_{\Theta_{R}} & -c_{\Theta_{R}}
\end{array}\right)=\left(\begin{array}{cc}
m_{\ell} & 0 \\
0 & M_{\mathcal{E}}
\end{array}\right), \\
-\left(\begin{array}{cc}
c_{\Theta_{\nu}^{\dagger}} & -s_{\Theta_{\nu}} \\
i s_{\Theta_{\nu}^{\dagger}} & i c_{\Theta_{\nu}}
\end{array}\right)\left(\begin{array}{cc}
0 & \lambda_{\nu} v / \sqrt{2} \\
\lambda_{\nu} v / \sqrt{2} & \lambda_{N} \mathcal{Y}_{N}
\end{array}\right)\left(\begin{array}{cc}
c_{\Theta_{\nu}^{\dagger}} & i s_{\Theta_{\nu}^{\dagger}} \\
-s_{\Theta_{\nu}} & i c_{\Theta_{\nu}}
\end{array}\right)=\left(\begin{array}{cc}
m_{\nu} & 0 \\
0 & \mathcal{M}_{N}
\end{array}\right),
\end{array}
$$

where $\Theta_{\nu}^{T}=\Theta_{\nu}$ has been used. Although these equations can be solved exactly, as done in ref. [15] for the quark case, the absence of a large Yukawa like that of the top quark seems to indicate that an expansion in $v / \mathcal{Y}$ is valid. In particular in the charged lepton sector, given eq. (2.14), the mixing terms are diagonal in flavour space $\left(\Theta_{\alpha \beta}=\delta_{\alpha \beta} \Theta_{\alpha \alpha}\right.$ and analogously for $\left.\Theta_{R}\right)$ :

$$
\begin{aligned}
\Theta & =\frac{\lambda_{E} v}{\sqrt{2} \lambda_{\mathcal{E}} \mathcal{Y}_{E}}+\mathcal{O}\left(\frac{v^{3}}{\mathcal{Y}_{E}^{3}}\right) \simeq \frac{\lambda_{E} v}{\sqrt{2} M_{\hat{\tau}}} \frac{m_{\ell}}{m_{\tau}}, \\
\Theta_{R} & =\frac{\mu_{E}}{\lambda_{\mathcal{E}} \mathcal{Y}_{E}}+\mathcal{O}\left(\frac{\mu_{E}^{3}}{\mathcal{Y}_{E}^{3}}\right) \simeq \frac{m_{\ell}^{2}}{m_{\tau} M_{\hat{\tau}}} \frac{1}{\Theta}=\frac{\mu_{E}}{M_{\hat{\tau}}} \frac{m_{\ell}}{m_{\tau}}, \\
\Theta_{\nu} & =\frac{\lambda_{\nu} v}{\sqrt{2} \lambda_{N} \mathcal{Y}_{N}}+\mathcal{O}\left(\frac{v^{3}}{\mathcal{Y}_{N}^{3}}\right) \simeq \frac{\lambda_{\nu} v}{\sqrt{2} \mathcal{M}_{N}} .
\end{aligned}
$$

In the case of $\mathcal{O}(1)$ dimensionless parameters considered here, the heavy $\mathcal{N}_{R}$ neutrino scale suppresses the mixing $\Theta_{\nu}$ which turns out to be $\mathcal{O}\left(10^{-10}\right)$; all the effects associated to $\Theta_{\nu}$ will thus be neglected in what follows.

After rotating to the mass basis, the fermion interaction Lagrangian is not diagonal, and in particular heavy-light couplings arise. It can be written as a sum of three terms:

$$
\mathscr{L}_{\psi \text {-int }}=\mathscr{L}_{\bar{\psi} \psi A^{\mathrm{SM}}}+\mathscr{L}_{\bar{\psi} \psi A^{F L}}+\mathscr{L}_{\bar{\psi} \psi \phi}
$$

The couplings to the SM gauge bosons can be casted in the conventional form,

$$
\mathscr{L}_{\bar{\psi} \psi A^{\mathrm{SM}}}=-e A_{\mu} J_{A}^{\mu}-\frac{g}{2 c_{W}} Z_{\mu} J_{Z}^{\mu}-\left(\frac{g}{\sqrt{2}} W_{\mu}^{+} J_{W}^{-\mu}+\text { h.c. }\right)
$$

with modified currents defined as

$$
\begin{aligned}
J_{\gamma}^{\mu}= & -\bar{e} \gamma^{\mu} e-\overline{\mathcal{E}} \gamma^{\mu} \mathcal{E} \\
J_{W}^{-\mu}= & \bar{\nu}_{L} U^{\dagger} \gamma^{\mu}\left(c_{\Theta} e_{L}+s_{\Theta} \mathcal{E}_{L}\right) \\
J_{Z}^{\mu}= & \bar{e} \gamma^{\mu}\left(-\left(c_{2 W}-s_{\Theta} s_{\Theta^{\dagger}}\right) P_{L}+2 s_{W}^{2} P_{R}\right) e-\overline{\mathcal{E}} \gamma^{\mu}\left(s_{\Theta^{\dagger}} s_{\Theta} P_{L}-2 s_{W}^{2}\right) \mathcal{E} \\
& +\bar{\nu}_{L} \gamma^{\mu} \nu_{L}-\left(\overline{\mathcal{E}}_{L} \gamma^{\mu} s_{\Theta^{\dagger}} c_{\Theta} e_{L}+\text { h.c. }\right),
\end{aligned}
$$

where $c_{W}\left(c_{2 W}\right)$ and $s_{W}$ stand for the cosine and sine of (twice) the Weinberg angle, respectively, and $P_{L, R}$ are the chirality projectors. Notice that the right-handed mixing $\Theta_{R}$ does not appear in the gauge interactions, because the SM quantum numbers of $\mathcal{E}_{R}$ 
and $e_{R}$ are the same. Most relevantly, as $\Theta$ is a diagonal matrix in flavour space as given in eq. (2.29), the transitions mediated by SM electroweak gauge bosons differ in the charged $\tau, \mu$ and $e$ sectors, with relative amplitudes given by $m_{\tau} / m_{\mu} / m_{e}$.

The interactions with flavour gauge bosons can be written as

$$
\mathscr{L}_{\bar{\psi} \psi A^{F L}}=-g_{\ell} \operatorname{Tr}\left(A_{\mu}^{\ell} J_{A^{\ell}}^{\mu}\right)-g_{E} \operatorname{Tr}\left(A_{\mu}^{E} J_{A^{E}}^{\mu}\right),
$$

where the currents are given in eq. (2.16). Notice that the difference between flavour and mass bases has been neglected in the previous expression, as that difference would only induce subleading effects in the observables of interest.

Finally, the couplings to the radial components of the scalar fields - that is, to the physical scalars - read, in the unitary gauge:

$$
\begin{aligned}
\mathscr{L}_{\bar{\psi} \psi \phi}= & \frac{-1}{\sqrt{2}}\left(\begin{array}{c}
\bar{e}_{L} \\
\overline{\mathcal{E}}_{L}
\end{array}\right)\left(\begin{array}{cc}
\left(\lambda_{E} c_{\Theta} h-\lambda_{\mathcal{E}} s_{\Theta} \phi_{E}\right) s_{\Theta_{R}} & \left(\lambda_{E} c_{\Theta} h-\lambda_{\mathcal{E}} s_{\Theta} \phi_{E}\right) c_{\Theta_{R}} \\
\left(\lambda_{\mathcal{E}} c_{\Theta^{\dagger}} \phi_{E}+\lambda_{E} s_{\Theta^{\dagger}} h\right) s_{\Theta_{R}} & \left(\lambda_{\mathcal{E}} c_{\Theta^{\dagger}} \phi_{E}+\lambda_{E} s_{\Theta^{\dagger}} h\right) c_{\Theta_{R}}
\end{array}\right)\left(\begin{array}{c}
e_{R} \\
\mathcal{E}_{R}
\end{array}\right) \\
& -\frac{\lambda_{\nu}}{\sqrt{2}} h \bar{\nu}_{L} \mathcal{N}_{R}-\frac{1}{2 \sqrt{2}} \overline{\mathcal{N}_{R}^{c}} \phi_{N} \mathcal{N}_{R}+\text { h.c. }
\end{aligned}
$$

The purely bosonic interactions follow from the Lagrangian in eq. (2.2) once the scalar potential is specified. The variables in this potential will determine the scalar mass spectrum which we do not examine in this work. However the scalar couplings to fermions given above do enjoy the flavour suppression characteristic of this model and will not disturb the flavour structure, as previously stated. Scalar excitation effects will be neglected in the phenomenological analysis that follows.

\subsection{Phenomenology}

The exchange of mirror charged leptons and $\mathrm{SU}(3)_{E}$ gauge bosons provides the dominant signals, as argued above, and it will be shown here that LUV signals are particularly prominent for $\tau$-related observables, while no charged lepton flavour violation (cLFV) is induced due to the preserved U(1) lepton number symmetry for each flavour: all modifications to SM couplings induced are flavour diagonal, as explained earlier. Flavour observables for the leading signals can be written in terms of only three independent parameters, which here are chosen to be

- The mixing parameter $\Theta .^{6}$

- The lightest mirror fermion mass $M_{\hat{\tau}}$.

- The norm $\left\|\mathcal{Y}_{E}^{-1}\right\|$, which is given approximately by its largest eigenvalue proportional to $m_{\tau}$, see eq. (2.26).

We determine next the bounds on these three parameters.

\footnotetext{
${ }^{6}$ Given one mixing angle, the other two are obtained from it by scaling.
} 
Bounds on the mixing parameters: the strongest bounds on $\Theta$ come from nonuniversality and non-unitarity of the PMNS matrix that follow from the (flavour diagonal) modifications of the couplings of leptons to $Z$ and $W$ bosons, eq. (2.32). The decay rate of the $Z$ boson to a pair of charged leptons (denoted by $l$ in the following equation) is now given by:

$$
\begin{aligned}
\Gamma\left(Z \rightarrow l^{-} l^{+}\right) & =\frac{g^{2} M_{Z}}{96 \pi c_{W}^{2}}\left(c_{2 W}^{2}+4 s_{W}^{4}-2 c_{2 W} s_{\Theta}^{2}\right)+\mathcal{O}\left(\Theta^{4}\right) \\
& =\Gamma_{\mathrm{SM}}\left(Z \rightarrow l^{-} l^{+}\right)\left(1-\frac{2 c_{2 W}}{c_{2 W}^{2}+4 s_{W}^{4}} \Theta_{l l}^{2}\right)+\mathcal{O}\left(\Theta^{4}\right),
\end{aligned}
$$

where the second line illustrates that the new contribution can only have a destructive interference with the SM one. The ratio of the branching ratios for the decay of $Z$ into $\tau^{+} \tau^{-}$and $e^{+} e^{-}$allows to extract explicitly the dependence on $\Theta_{\tau \tau}$,

$$
\frac{\operatorname{Br}\left(Z \rightarrow \tau^{+} \tau^{-}\right)}{\operatorname{Br}\left(Z \rightarrow e^{+} e^{-}\right)}-1 \simeq-2.14 \Theta_{\tau \tau}^{2} .
$$

The experimental bound [24] on the observable on the left hand side of this expression leads to a strong limit on $\Theta$ :

$$
\frac{\operatorname{Br}\left(Z \rightarrow \tau^{+} \tau^{-}\right)}{\operatorname{Br}\left(Z \rightarrow e^{+} e^{-}\right)}-1=0.0019 \pm 0.0032 \Longrightarrow\left|\Theta_{\tau \tau}\right|=\frac{\lambda_{E} v}{\sqrt{2} M_{\hat{\tau}}} \leq 4.5 \times 10^{-2},
$$

where the bound has been rescaled to the 95\% CL assuming a gaussian behaviour. In consequence, using eq. (2.29),

$$
\left|\Theta_{\mu \mu}\right| \leq 2.7 \times 10^{-3}, \quad\left|\Theta_{e e}\right| \leq 1.3 \times 10^{-5} .
$$

At this point it is pertinent to ask whether the persistent anomalies in the decay of $B$ meson into $K$ and $K^{*}$ bosons [37, 38] could be induced by the modifications to $Z$-fermion couplings just discussed, as precisely they tend to diminish the decay rate into $\mu$ and $\tau$ leptons while the electronic channels are almost uncorrected; this could happen for instance via a Z-penguin loop attached to the quark legs and/or through the equivalent mechanisms when gauging flavour in the quark sector [15]. Nevertheless, the bounds just set on $\Theta_{\mu \mu}$ are too strong compared with the experimental anomaly which, if confirmed, would require $\mathcal{O}(1)$ corrections.

Similar bounds on $\Theta$ can be inferred from the analysis of non-unitary contributions to the diagonal elements of the PMNS matrix $U$, to which other observables contribute. The leptonic mixing matrix is now corrected by

$$
\tilde{U} \equiv \cos \Theta U, \quad\left(\tilde{U} \tilde{U}^{\dagger}\right)_{\alpha \beta}-\delta_{\alpha \beta} \simeq-\Theta_{\alpha \beta}^{2}=-\frac{\lambda_{E}^{2} v^{2}}{2 M_{\hat{\tau}}^{2}} \delta_{\alpha \beta} \frac{m_{\alpha}^{2}}{m_{\tau}^{2}},
$$

and in consequence the most stringent bound stems again from the $\tau \tau$ entry; bounds on the diagonal entries can be derived from a global fit to lepton universality and precision electroweak observables [39], yielding

$$
\left|\Theta_{\tau \tau}\right| \leq 7.5 \times 10^{-2},
$$


at 95\% CL. An alternative bayesian global fit can be found in ref. [40] resulting in $\left|\Theta_{\tau \tau}\right| \leq 7.6 \times 10^{-2}$.

Bounds on $\boldsymbol{M}_{\hat{\tau}}$ : the heavy-light fermion mixing is controlled by the Yukawa couplings, see eq. (2.6), and in consequence the lightest fermion of the heavy spectrum - the mirror tau - will decay predominantly to channels involving longitudinal gauge bosons $W_{L}$ and $Z_{L}$ and the Higgs particle, provided $\hat{\tau}$ is heavy enough,

$$
\Gamma\left(\hat{\tau} \rightarrow Z_{L} \tau\right)=\frac{\lambda_{E}^{2} M_{\hat{\tau}}}{64 \pi}, \quad \Gamma\left(\hat{\tau} \rightarrow W_{L} \nu_{\tau}\right)=\frac{\lambda_{E}^{2} M_{\hat{\tau}}}{32 \pi}, \quad \Gamma(\hat{\tau} \rightarrow h \tau)=\frac{\lambda_{E}^{2} M_{\hat{\tau}}}{64 \pi} .
$$

The $\hat{\tau}$ fermion is electrically charged and it would thus be copiously pair-produced in $e^{+} e^{-}$colliders via photon exchange, if sufficiently light. The lack of evidence for new resonances and for charged heavy leptons in LEP data [41] sets a constraint

$$
M_{\hat{\tau}} \gtrsim 100.8 \mathrm{GeV} \quad \text { at } 95 \% \mathrm{CL},
$$

a bound that does not depend on the mixing parameter $\Theta$. The LHC can provide stronger constraints on the mass of the mirror taus. The most sensitive channel would involve pair production of $\hat{\tau}$ via neutral current or photon exchange and their subsequent decay to $\tau+Z$ with $\sim 25 \%$ branching ratio. To the best of our knowledge such a search has not been performed yet. Related searches for SUSY chargino pair production and their decay to $W$ plus missing energy (neutralino) currently constrain chargino masses to be above $\sim 620 \mathrm{GeV}$ [42]. The decay of the $\hat{\tau}$ to $W+\nu$ would lead to a similar final state, although with somewhat different kinematics. Thus, similar constraints are expected to hold for the $\hat{\tau}$, however a dedicated search that directly applies to this scenario is still missing and needed.

Bounds on $\left\|\mathcal{Y}_{\boldsymbol{E}}^{-1}\right\|$ : eq. (2.42) can be translated into a limit on the flavon vev, applying eq. (2.9),

$$
\left\|\mathcal{Y}_{E}^{-1}\right\|=\frac{\lambda_{\mathcal{E}}}{M_{\hat{\tau}}}\left(1+\mathcal{O}\left(m_{\mu}^{2} / m_{\tau}^{2}\right)\right)<0.01 \lambda_{\mathcal{E}} \mathrm{GeV}^{-1}, \quad \text { at } \quad 95 \% \mathrm{CL}
$$

Moreover, bounds on $\left\|\mathcal{Y}_{E}^{-1}\right\|$ independent from $\lambda_{\mathcal{E}}$ can be extracted from the limits on four-lepton interactions induced by the exchange of $\mathrm{SU}(3)_{E}$ gauge bosons among right-handed charged SM leptons. Integrating out those $A_{\mu}^{E}$ gauge bosons results in effective low-energy couplings of the form

$$
-\frac{c_{E}^{\alpha \beta \kappa \rho}}{2}\left\|\mathcal{Y}_{E}^{-1}\right\|^{2}\left(\bar{e}_{R}^{\alpha} \gamma_{\mu} e_{R}^{\beta}\right)\left(\bar{e}_{R}^{\kappa} \gamma^{\mu} e_{R}^{\rho}\right),
$$

which do not exhibit a dependence on the coupling constant $g_{E}$. The coefficient $c_{E}$ encodes a specific flavour-conserving suppression:

$$
c_{E}^{\alpha \beta \kappa \rho}=\frac{m_{\alpha}^{2} m_{\kappa}^{2}}{\sum_{\gamma} m_{\gamma}^{2}}\left[\delta_{\alpha \rho} \delta_{\beta \kappa} \frac{1}{m_{\alpha}^{2}+m_{\kappa}^{2}}-\delta_{\alpha \beta} \delta_{\kappa \rho} \frac{1}{2 \sum_{\gamma} m_{\gamma}^{2}}\right],
$$


where the last term would be absent if gauging the full $\mathrm{U}(3)_{E}$. This expression is (treelevel) exact up to $\mathcal{Y}_{E} / \mathcal{Y}_{N}$ corrections as opposed to the approximate mass formula in eq. (2.25). Considering specifically a process involving two electrons (denoted here by $\left.e_{R}^{1}\right)$ and two other generic charged leptons $e_{R}^{\alpha}$, eq. (2.44) becomes $^{7}$

$$
\left\|\mathcal{Y}_{E}^{-1}\right\|^{2} \frac{m_{e}^{2}}{m_{\tau}^{2}}\left(1+\delta_{\alpha 1}\right)\left(\frac{2 m_{\tau}^{2} m_{\alpha}^{2}-m_{\alpha}^{2}\left(m_{e}^{2}+m_{\alpha}^{2}\right)}{2\left(m_{e}^{2}+m_{\alpha}^{2}\right) m_{\tau}^{2}}\right)\left(\bar{e}_{R}^{1} \gamma^{\mu} e_{R}^{1}\right)\left(\bar{e}_{R}^{\alpha} \gamma^{\mu} e_{R}^{\alpha}\right)
$$

where $\sum m_{\beta}^{2} \simeq m_{\tau}^{2}$ has been used. These operators are suppressed by an extra $\sim$ $m_{e}^{2} / m_{\tau}^{2}$ factor with respect to the case where no flavour symmetry is implemented [43]. Equivalently, it can be argued that the effective scale associated to the new physics responsible for these processes can be $m_{\tau} / m_{e}$ smaller than in the case without flavour symmetry protection, in a pattern reminiscent of MFV as expected. The bounds stemming from LEP data [44] on four-fermion interactions involving two electrons can thus be translated into $95 \% \mathrm{CL}$ constraints on $\left\|\mathcal{Y}_{E}^{-1}\right\|$ :

$$
\begin{array}{lll}
e^{+} e^{-} \rightarrow e^{+} e^{-} & \Longrightarrow & \left\|\mathcal{Y}_{E}^{-1}\right\|<0.41(0.44) \mathrm{GeV}^{-1}, \\
e^{+} e^{-} \rightarrow \mu^{+} \mu^{-} & \Longrightarrow & \left\|\mathcal{Y}_{E}^{-1}\right\|<0.37(0.30) \mathrm{GeV}^{-1}, \\
e^{+} e^{-} \rightarrow \tau^{+} \tau^{-} & \Longrightarrow & \left\|\mathcal{Y}_{E}^{-1}\right\|<0.57(0.57) \mathrm{GeV}^{-1},
\end{array}
$$

where the first (second) value is for destructive (constructive) interference with the SM contributions. These constraints are weak but complementary to that in eq. (2.43) since they are independent from $\lambda_{\mathcal{E}}$.

Stronger bounds on $\left\|\mathcal{Y}_{E}^{-1}\right\|$ can be inferred from present data on other flavour conserving processes such as magnetic moments, to which the flavour $\mathrm{SU}(3)_{E}$ gauge bosons may contribute. Defining as is customary the muon anomalous magnetic moment, $a_{\mu}$, as the coefficient of the muon dipole operator in the effective Lagrangian [45]

$$
\mathscr{L}_{(g-2)_{\mu}} \equiv-\frac{a_{\mu} e}{4 m_{\mu}} \bar{\mu} \sigma_{\rho \delta} \mu F^{\rho \delta}+\text { h.c. },
$$

it is easy to see that penguin diagrams mediated by the $\mathrm{SU}(3)_{E}$ flavour gauge bosons induce a correction of the form

$$
\delta a_{\mu}=-\frac{m_{\mu}^{2}}{12 \pi^{2}} \sum \frac{g_{E}^{2}}{M_{A_{a}^{E}}^{2}}\left(\hat{T}^{a} \cdot \hat{T}^{a}\right)_{\mu \mu} \simeq-\frac{3}{4} \frac{m_{\mu}^{4}}{6 \pi^{2} m_{\tau}^{2}}\left\|\mathcal{Y}_{E}^{-1}\right\|^{2},
$$

where the Casimir factor of $3 / 4$ results from the $\mathrm{SU}(2)_{\mu-\tau}$ quasi-degeneracy among the lightest gauge bosons. Note that the sign of the contribution obtained is negative, ${ }^{8}$ as the SM one, and therefore it does not help to relax the tension between the SM prediction and the experimental determination, $\Delta a_{\mu} \equiv a_{\mu}^{\mathrm{Exp}}-a_{\mu}^{\mathrm{SM}}=287(63)(49) \times 10^{-11}$ [24]. However,

\footnotetext{
${ }^{7}$ In eqs. (2.45) and (2.46) $m_{1}=m_{e}, m_{2}=m_{\mu}$ and $m_{3}=m_{\tau}$.

${ }^{8}$ The sign of the contribution is negative because the lightest gauge bosons couple only to the righthanded leptons. For a detailed analysis of the role of the chirality of the couplings to leptons in the $g-2$ contributions see, e.g., ref. [23].
} 
requiring that the flavour correction does not increase the present tension beyond $5 \sigma$, the following bound follows:

$$
\left\|\mathcal{Y}_{E}^{-1}\right\| \leq 0.047 \mathrm{GeV}^{-1}, \quad \text { or equivalently } \quad\left\|\mathcal{Y}_{E}\right\| \geq 7.4 \times 10^{4} \mathrm{GeV} .
$$

Note that, unlike for the other constraints discussed in this section, a 95\% CL has not been adopted in this bound since the SM prediction itself already presents a stronger disagreement with current data.

It is interesting to translate the bounds on $\left\|\mathcal{Y}_{E}\right\|$ into a limit on the flavour gauge boson mass scale. Eq. (2.50) translates into a limit on the mass of the lightest gauge bosons $A^{E, 3}$, $A^{E, 6}, A^{E, 7}$ given by

$$
M_{A^{E, i}} \gtrsim 2.5 \times 10^{2} g_{E} \mathrm{GeV},
$$

as a function of the gauge flavour coupling $g_{E}$. In the case of the illustrative benchmark spectrum considered in figure 2, the lightest flavour gauge bosons have masses of $\mathcal{O}(10) \mathrm{TeV}$, largely satisfying the bounds obtained in this section assuming a perturbative weak regime for the new gauge sectors.

\section{Gauged lepton flavour Seesaw model: $\mathrm{SU}(3)_{\ell} \times \mathrm{SU}(3)_{E} \times \mathrm{SO}(3)_{N}$}

In the context of the type I Seesaw theory with three degenerate right-handed neutrinos $N_{R}$, the maximal flavour symmetry group of the Lagrangian in the limit of vanishing masses for the three known fermion families is $\mathrm{U}(3)_{\ell} \times \mathrm{U}(3)_{E} \times O(3)_{N}$. The latter is the symmetry exhibited by the kinetic terms plus heavy degenerate right-handed neutrinos,

$$
\mathcal{L}=i \bar{\ell}_{L} \not D \ell_{L}+i \bar{e}_{R} \not D e_{R}+i \bar{N}_{R} \not \partial N_{R}+\frac{1}{2}\left\{\mu_{\mathrm{LN}} \overline{N_{R}{ }^{c}} N_{R}+\text { h.c. }\right\} .
$$

As earlier stated, we focus on flavour effects and restrain here to gauging the nonabelian factors $\mathrm{SU}(3)_{\ell} \times \mathrm{SU}(3)_{E} \times \mathrm{SO}(3)_{N}$ only. The field content that needs to be added then in order to cancel gauge anomalies is identical to that in the previous model, since triangle diagrams cancel for $\mathrm{SO}(3)_{N}$ and the $N_{R}$ fermions are singlets under the SM gauge symmetry. The fermion spectrum is summarized in table 2 ; note that the quantum numbers for $\mathcal{Y}_{N}$ differ from those in the previous section.

Using again and until further notice unprimed fields to denote flavour eigenstates, the Lagrangian describing the model can be written as that in eq. (2.2), where now $\mathscr{L}_{Y}$ encodes both Yukawa interactions and Majorana mass terms,

$$
\begin{aligned}
\mathscr{L}_{Y}= & \lambda_{E} \bar{\ell}_{L} H \mathcal{E}_{R}+\mu_{E} \overline{\mathcal{E}}_{L} e_{R}+\lambda_{\mathcal{E}} \overline{\mathcal{E}}_{L} \mathcal{Y}_{E} \mathcal{E}_{R} \\
& +\lambda_{\nu} \bar{\ell}_{L} \tilde{H} \mathcal{N}_{R}+\lambda_{N} \overline{N_{R}^{c}} \mathcal{Y}_{N} \mathcal{N}_{R}+\frac{\mu_{\mathrm{LN}}}{2}{\overline{N_{R}}}^{c} N_{R}+\text { h.c. }
\end{aligned}
$$

where again all overall constants, i.e., $\lambda$ 's and $\mu$ 's, can be made real via chiral rotations. The only source of $\mathrm{CP}$ violation lies then in the non-trivial flavour structure of the vevs of the scalar fields $\mathcal{Y}_{E}$ and $\mathcal{Y}_{N}$. The charged lepton mass matrix inferred from this Lagrangian is identical to that in eq. (2.8), and in consequence the particle spectrum and phenomenology of the $\mathrm{SU}(3)_{E}$ sector (gauge bosons and mirror charged leptons) matches the description 


\begin{tabular}{|c|ccccc|}
\hline & $\mathrm{SU}(2)_{L}$ & $\mathrm{U}(1)_{Y}$ & $\mathrm{SU}(3)_{\ell}$ & $\mathrm{SU}(3)_{E}$ & $\mathrm{SO}(3)_{N}$ \\
\hline$\ell_{L} \equiv\left(\nu_{L}, e_{L}\right)$ & 2 & $-1 / 2$ & 3 & 1 & 1 \\
$e_{R}$ & 1 & -1 & 1 & 3 & 1 \\
$N_{R}$ & 1 & 0 & 1 & 1 & 3 \\
\hline $\mathcal{E}_{R}$ & 1 & -1 & 3 & 1 & 1 \\
$\mathcal{E}_{L}$ & 1 & -1 & 1 & 3 & 1 \\
$\mathcal{N}_{R}$ & 1 & 0 & 3 & 1 & 1 \\
\hline $\mathcal{Y}_{E}$ & 1 & 0 & $\overline{3}$ & 3 & 1 \\
$\mathcal{Y}_{N}$ & 1 & 0 & $\overline{3}$ & 1 & 3 \\
\hline
\end{tabular}

Table 2. Transformation properties of SM fields, of (flavour) mirror fields and of flavons under the $\mathrm{EW}$ group and $\mathrm{SU}(3)_{\ell} \times \mathrm{SU}(3)_{E} \times \mathrm{SO}(3)_{N}$.

given in the previous section. In contrast, the particle spectrum and phenomenology of the $\mathrm{SU}(3)_{\ell}$ and $\mathrm{SO}(3)_{N}$ sectors (gauge bosons and heavy neutral fermions) will now depend on three fundamental scales: the vevs of $\mathcal{Y}_{E}$ and $\mathcal{Y}_{N}$ and the lepton number parameter $\mu_{\mathrm{LN}}$. Note that now the LN and flavour scales are distinct; for instance for $\mu_{\mathrm{LN}}=0$, there will still be be physical leptonic mixing and flavour effects associated to $\mathcal{Y}_{N}$. The neutral fermions mass matrix in the Lagrangian eq. (3.2) (in the $\left\{\ell^{c}, \mathcal{N}_{R}, N_{R}\right\}$ basis),

$$
\frac{1}{2}\left(\begin{array}{ccc}
0 & \lambda_{\nu} v / \sqrt{2} & 0 \\
\lambda_{\nu} v / \sqrt{2} & 0 & \lambda_{N} \mathcal{Y}_{N}^{T} \\
0 & \lambda_{N} \mathcal{Y}_{N} & \mu_{\mathrm{LN}}
\end{array}\right)+\text { h.c. }
$$

is typical of inverse Seesaw scenarios [46-48], in which generically that separation of the two scales holds. Eq. (3.3) immediately suggests two interesting limiting regimes for the parameters $\mathcal{Y}_{N}$ and $\mu_{\mathrm{LN}}$ :

$\boldsymbol{\mu}_{\mathrm{LN}} \gg \mathcal{Y}_{N}$ : in this limit the $N_{R}$ fields would decouple producing an effective mass term for the $\mathcal{N}_{R}$ of the form $\mathcal{Y}_{N} \mathcal{Y}_{N}^{T} / \mu_{\mathrm{LN}}$. The basic type I Lagrangian of the previous model is recovered, albeit with the $(2,2)$ entry of the neutral mass matrix in eq. (2.8) replaced by that effective mass.

$\mathcal{Y}_{\boldsymbol{N}} \gg \boldsymbol{\mu}_{\mathrm{LN}}$ : an approximate $\mathrm{U}(1)_{\mathrm{LN}}$ symmetry holds in this limit, as often explored within low-scale inverse Seesaw scenarios [49-51]. $N_{R}^{c}$ and $\mathcal{N}_{R}$ would form pseudoDirac pairs and the light neutrino masses will be suppressed by a factor $\mu_{\mathrm{LN}} /\left(\lambda_{N} \mathcal{Y}_{N}\right)$ with respect to those for the basic type I Seesaw in eq. (2.8).

The second limit leads to new phenomenology and will be the focus of the rest of the section. The interplay between $\mathcal{Y}_{E}$ and $\mathcal{Y}_{N}$ will determine the spectrum and the phenomenology of the flavour gauge bosons and will be discussed next. 


\subsection{Fermion spectrum and interactions: $\mathcal{Y}_{N} \gg \mu_{\mathrm{LN}}$ case}

It is possible to expect in this model measurable signals of lepton-flavour violation, precisely because the LN parameter $\left(\mu_{\mathrm{LN}}\right)$ and lepton flavour violation scale $\left(\left\|\mathcal{Y}_{N}\right\|\right)$ are independent and the latter is not strongly constrained by the tiny value of light neutrino masses. By the same token, the mirror neutral fermions - determined by $\left\|\mathcal{Y}_{N}\right\|$ - are now allowed to be much lighter than in the gauged-flavour SM discussed in section 2, see eq. (2.13), and close to those of the charged lepton mirror fermions. Indeed, in the $\mu_{\mathrm{LN}} \ll \mathcal{Y}_{N}$ limit the singlet fermions $\mathcal{N}_{R}$ and $N_{R}^{c}$ form Dirac pairs of mass

$$
\mathcal{M}_{N} \simeq \lambda_{N} \mathcal{Y}_{N}
$$

where we neglected $\lambda_{\nu} v$ contributions and the mass splitting in quasi-Dirac fields is given by $\mu_{\mathrm{LN}}$, while the three light neutrinos acquire Majorana masses suppressed by the LN scale, which does not carry flavour structure, ${ }^{9}$

$$
m_{\nu}=\frac{v^{2}}{2} \frac{C_{\nu}}{\Lambda_{\mathrm{LN}}} \simeq \frac{v^{2}}{2} \frac{\lambda_{\nu}^{2}}{\lambda_{N}^{2}} \frac{1}{\mathcal{Y}_{N}} \mu_{\mathrm{LN}} \frac{1}{\mathcal{Y}_{N}^{T}} .
$$

The lightness of neutrino masses can be thus attributed to a small $\mu_{\mathrm{LN}}$ instead of a very large $\left\|\mathcal{Y}_{N}\right\|$ (needed in the previous section): this is a technically natural solution as $\mu_{\mathrm{LN}}$ is protected by the approximate $\mathrm{U}(1)_{\mathrm{LN}}$ symmetry. In consequence, $\left\|\mathcal{Y}_{N}\right\|$ can now be of the order of the electroweak scale or even smaller, resulting in putatively observable signals of lepton-flavour violation mediated by flavour gauge bosons of the $\mathrm{SU}(3)_{\ell} \times \mathrm{SO}(3)_{N}$ sector (see further below) independently of the value of light neutrino masses.

Note that, as in the gauged-flavour SM in section 2, the mirror lepton mass matrices are linearly proportional to the flavon vevs $\mathcal{Y}_{E}$ (eq. (2.9)) and $\mathcal{Y}_{N}$ (eq. (3.4)), and the mass of the SM charged leptons is inversely proportional to $\mathcal{Y}_{E}$ (eq. (2.10)); in contrast, the light neutrino masses exhibit now a quadratic inverse dependence on $\mathcal{Y}_{N}$, eq. (3.5). From this equation a parametrization equivalent to that of Casas-Ibarra [52] can be introduced:

$$
\mathcal{Y}_{N}=\frac{v}{\sqrt{2}} \frac{\lambda_{\nu}}{\lambda_{N}} R \sqrt{\frac{\mu_{\mathrm{LN}}}{m_{\nu}^{\text {diag }}}} U^{\dagger},
$$

where $U$ is the PMNS matrix and $m_{\nu}^{\text {diag }}$ is the diagonal matrix of light neutrino masses $m_{\nu_{i}}$,

$$
m_{\nu}^{\operatorname{diag}} \equiv\left(m_{\nu_{1}}, m_{\nu_{2}}, m_{\nu_{3}}\right)
$$

and $R$ is an orthogonal complex matrix. The latter can be parametrized in general as the exponential of the anti-symmetric Gell-Mann matrices with complex coefficients, although in the case discussed an $\mathrm{SO}(3)_{N}$ transformation allows to remove the imaginary part of these coefficients,

$$
R=e^{\eta_{i} T^{i}}, \quad R R^{T}=\mathbb{1}, \quad R=R^{\dagger},
$$

where $\eta_{i}$ are three real parameters and the matrices $T^{i}$ denote the set of three generators $\left\{T^{2}, T^{5}, T^{7}\right\}$.

\footnotetext{
${ }^{9}$ The effective LN scale here is thus $\Lambda_{\mathrm{LN}} \sim\left\|\mathcal{M}_{N}\right\|^{2} / \mu_{\mathrm{LN}}$, as usual in inverse Seesaw constructions, while the scale suppressing flavour effects is $\left\|\mathcal{M}_{N}\right\|$.
} 
In the rest of this section, and in analogy with eq. (2.27), we revert again to the notation in which flavour eigenstates are denoted by primed fields while unprimed ones stand for the mass eigenstates. In the limit of vanishing $\mu_{\mathrm{LN}}$, which will be assumed from now on, the mass term for neutrinos coming from eq. (3.2) after symmetry breaking reduces to

$$
\left(\lambda_{\nu} \overline{\nu_{L}^{\prime}} v / \sqrt{2}+\lambda_{N} \overline{N_{R}^{\prime c}} \mathcal{Y}_{N}\right) \mathcal{N}_{R}^{\prime}+\text { h.c. }=-\bar{N}_{R}^{c} \mathcal{M}_{N} \mathcal{N}_{R}+\text { h.c. },
$$

and therefore a unitary rotation among only the $\nu_{L}$ and $N_{R}$ fields suffices to diagonalize the mass matrix:

$$
\left(\begin{array}{c}
\nu_{L}^{\prime} \\
N_{R}^{\prime c}
\end{array}\right)=\left(\begin{array}{cc}
c_{\Theta_{\nu}} & s_{\Theta_{\nu}} \\
-s_{\Theta_{\nu}^{\dagger}} & c_{\Theta_{\nu}^{\dagger}}
\end{array}\right)\left(\begin{array}{c}
\nu_{L} \\
N_{R}^{c}
\end{array}\right),
$$

where $\Theta_{\nu}$ is as given in eq. (2.29) and we simultaneously define $\mathcal{N}_{R}^{\prime}=-\mathcal{N}_{R}$ in order to recover the usual sign for the Dirac mass term of the heavy states, and in accordance with the definitions in the gauged-flavour SM, eqs. (2.27) and (2.34).

Interactions with SM gauge bosons. $\mathcal{Y}_{N}$ introduces new flavour non-conserving transitions, associated to the extra fermionic states and parameterized by $\Theta_{\nu}$. The flavour changing and light-heavy mixing effects can then be written in the mass basis as in eq. (2.31), where now

$$
\begin{aligned}
J_{\gamma}^{\mu}= & -\bar{e} \gamma^{\mu} e-\overline{\mathcal{E}} \gamma^{\mu} \mathcal{E} \\
J_{W}^{-\mu}= & \bar{\nu}_{L} \gamma^{\mu} U^{\dagger} c_{\Theta_{\nu}}\left(c_{\Theta} e_{L}+s_{\Theta} \mathcal{E}_{L}\right)+\overline{N_{R}^{c}} \gamma^{\mu} s_{\Theta_{\nu}}^{\dagger}\left(c_{\Theta} e_{L}+s_{\Theta} \mathcal{E}_{L}\right), \\
J_{Z}^{\mu}= & \bar{e} \gamma^{\mu}\left(-\left(c_{2 W}-s_{\Theta} s_{\Theta^{\dagger}}\right) P_{L}+2 s_{W}^{2} P_{R}\right) e-\overline{\mathcal{E}} \gamma^{\mu}\left(s_{\Theta^{\dagger}} s_{\Theta} P_{L}-2 s_{W}^{2}\right) \mathcal{E}-\left(\overline{\mathcal{E}}_{L} \gamma^{\mu} s_{\Theta^{\dagger}} c_{\Theta} e_{L}+\text { h.c. }\right) \\
& +\bar{\nu}_{L} \gamma^{\mu} c_{\Theta_{\nu}}^{2} \nu_{L}+\bar{N}_{R} \gamma^{\mu} s_{\Theta_{\nu}^{\dagger}} s_{\Theta_{\nu}} N_{R}+\left(\bar{\nu}_{L} \gamma^{\mu} c_{\Theta_{\nu}} s_{\Theta_{\nu}} N_{R}^{c}+\text { h.c. }\right) .
\end{aligned}
$$

Note that the PMNS matrix appearing in $W$ couplings is given by the product $U^{\dagger} c_{\Theta_{\nu}} c_{\Theta}$, with $U$ being its unitary part and $\Theta_{\nu}$ and $\Theta$ encoding deviations from unitarity. The expressions for the mixing angles equal those in the previous section, eq. (2.29).

Scalar interactions. Using the definitions in eq. (2.7) for the scalar excitations, the generalized Yukawa interactions read for vanishing $\mu_{\mathrm{LN}}$ :

$$
\begin{aligned}
& \mathscr{L}_{\bar{\psi} \psi \phi}=\frac{-1}{\sqrt{2}}\left(\begin{array}{c}
\bar{e}_{L} \\
\overline{\mathcal{E}}_{L}
\end{array}\right)\left(\begin{array}{cc}
\left(\lambda_{E} c_{\Theta} h-\lambda_{\mathcal{E}} s_{\Theta} \phi_{E}\right) s_{\Theta_{R}} & \left(\lambda_{E} c_{\Theta} h-\tilde{\lambda}_{\mathcal{E}} s_{\Theta} \phi_{E}\right) c_{\Theta_{R}} \\
\left(\lambda_{\mathcal{E}} c_{\Theta^{\dagger}} \phi_{E}+\lambda_{E} s_{\Theta^{\dagger}} h\right) s_{\Theta_{R}} & \left(\lambda_{\mathcal{E}} c_{\Theta^{\dagger}} \phi_{E}+\lambda_{E} s_{\Theta^{\dagger}} h\right) c_{\Theta_{R}}
\end{array}\right)\left(\begin{array}{c}
e_{R} \\
\mathcal{E}_{R}
\end{array}\right) \\
& -\frac{\lambda_{\nu}}{\sqrt{2}} h\left(\bar{\nu}_{L} c_{\Theta_{\nu}}+\overline{N_{R}^{c}} s_{\Theta_{\nu}^{\dagger}}\right) \mathcal{N}_{R}-\frac{\lambda_{N}}{\sqrt{2}}\left(\overline{N_{R}^{c}} c_{\Theta_{\nu}^{\dagger}}-\bar{\nu}_{L} s_{\Theta_{\nu}}\right) \phi_{N} \mathcal{N}_{R}+\text { h.c. . }
\end{aligned}
$$

In this model there are $18+18-19=17$ scalars $\phi_{E}$ and $\phi_{N},{ }^{10}$ which are fluctuations around the 6 mixing parameters, 6 masses, 3 variables in the orthogonal self-hermitian matrix $R$ and two phases in $\mathrm{U}(1)_{\ell}$ and $\mathrm{U}(1)_{E}$. Their effects are strongly suppressed [15] and will not be further discussed.

\footnotetext{
${ }^{10}$ Among the 36 real degrees of freedom of the two $3 \times 3$ complex matrices $\mathcal{Y}_{E}$ and $\mathcal{Y}_{N}$, 19 become the longitudinal components of the 19 flavour gauge bosons of the model.
} 
Were the extra neutral states $N$ lighter than the Higgs boson, the following decay channel would open:

$$
\Gamma(h \rightarrow N \nu)=\frac{\lambda_{\nu}^{2}}{16 \pi} M_{h}\left(1-\frac{M_{N}^{2}}{M_{h}^{2}}\right),
$$

where $N$ stands here for the generic mass eigenstates. The $N$ fields will in turn be unstable and decay to lighter charged fermions and neutrinos via the interaction in eq. (3.11), with a pattern that depends strongly on $M_{N}$ and $\Theta_{\nu}$, potentially leading to new visible Higgs decays, displaced vertices or contributions to the invisible decay. Additional bounds would then apply; we will not further consider this case of heavy neutrinos lighter than the Higgs particle.

Flavour gauge boson spectrum and interactions. Additional flavour non-conserving effects can be induced by flavour gauge bosons, $A_{\mu}^{\ell}$. Indeed, the theory contains nineteen flavour gauge bosons whose Lagrangian reads

$$
\begin{aligned}
& \sum_{I} \operatorname{Tr}\left(A_{\mu}^{I} \partial^{2} A^{I, \mu}\right)+\operatorname{Tr}\left\{\left(g_{E} A_{\mu}^{E} \mathcal{Y}_{E}-g_{\ell} \mathcal{Y}_{E} A_{\mu}^{\ell}\right)\left(g_{E} \mathcal{Y}_{E}^{\dagger} A^{E, \mu}-g_{\ell} A^{\ell, \mu} \mathcal{Y}_{E}^{\dagger}\right)\right\} \\
& \quad+\operatorname{Tr}\left\{\left(g_{N} A_{\mu}^{N} \mathcal{Y}_{N}-g_{\ell} \mathcal{Y}_{N} A_{\mu}^{\ell}\right)\left(g_{N} \mathcal{Y}_{N}^{\dagger} A^{N, \mu}-g_{\ell} A^{\ell, \mu} \mathcal{Y}_{N}^{\dagger}\right)\right\}-\sum_{I} g_{I} \operatorname{Tr}\left(A_{\mu}^{I} J_{A_{I}}^{\mu}\right)
\end{aligned}
$$

where cubic and quartic gauge boson interactions are not shown as they will play no role in the phenomenological analysis below. In eq. (3.14) the ensemble of fields $A_{\mu}^{I}, I=\ell, E, N$, is treated as a traceless hermitian matrix and the currents are defined as matrices in flavour space, with the currents $J_{A^{\ell}}^{\mu}$ and $J_{A^{E}}^{\mu}$ as defined in eq. (2.16) and the $\mathrm{SO}(3)_{N}$ current given by

$$
\left[J_{A^{N}}^{\mu}\right]_{i j}=\frac{1}{2}\left(\bar{N}_{R}^{j} \gamma^{\mu} N_{R}^{i}-\bar{N}_{R}^{i} \gamma^{\mu} N_{R}^{j}\right)
$$

The EOM resulting from eq. (3.14) for $A_{\mu}^{E}$ is identical to that in eq. (2.18), while for $A_{\mu}^{\ell}$ and $A_{\mu}^{N}$ they are given by

$$
\begin{gathered}
\partial^{2} A_{\mu}^{\ell}-g_{E} g_{\ell} \mathcal{Y}_{E}^{\dagger} A_{\mu}^{E} \mathcal{Y}_{E}-g_{N} g_{\ell} \mathcal{Y}_{\nu}^{\dagger} A_{\mu}^{N} \mathcal{Y}_{N}+\frac{g_{\ell}^{2}}{2}\left\{\mathcal{Y}_{E}^{\dagger} \mathcal{Y}_{E}+\mathcal{Y}_{N}^{\dagger} \mathcal{Y}_{N}, A_{\mu}^{\ell}\right\}-\frac{g_{\ell}}{2} J_{\mu}^{A^{\ell}}=\frac{1}{n_{g}} \operatorname{Tr}(\text { L.H.S. }) \mathbb{1}, \\
\partial^{2} A_{\mu}^{N}+\frac{g_{N}^{2}}{4}\left\{\mathcal{Y}_{N} \mathcal{Y}_{N}^{\dagger}+\mathcal{Y}_{N}^{*} \mathcal{Y}_{N}^{T}, A_{\mu}^{N}\right\}-\frac{g_{\ell} g_{N}}{2}\left(\mathcal{Y}_{N} A_{\mu}^{\ell} \mathcal{Y}_{N}^{\dagger}-\mathcal{Y}_{N}^{*}\left(A_{\mu}^{\ell}\right)^{T} \mathcal{Y}_{N}^{T}\right)-\frac{g_{N}}{2} J_{\mu}^{A^{N}}=0,
\end{gathered}
$$

where $n_{g}=3$. Eq. (3.14) can be alternatively written in a compact matrix notation arranging the flavour gauge bosons in an array $\chi_{\mu}^{a}=\left(A_{\mu}^{\ell, 1}, \ldots, A_{\mu}^{\ell, 8}, A_{\mu}^{E, 1}, \ldots, A_{\mu}^{E, 8}, A_{\mu}^{N, 1}, \ldots, A_{\mu}^{N, 3}\right)$ :

$$
\mathscr{L}_{\text {gauge }}=-\frac{1}{2} \sum_{I=\ell, E, N} \operatorname{Tr}\left(F_{\mu \nu}^{I} F_{I}^{\mu \nu}\right)+\frac{1}{2} \sum_{a, b=1}^{19} \chi_{\mu}^{a}\left(M_{A}^{2}\right)_{a b} \chi^{b, \mu}-\sum_{I=\ell, E, N} g_{I} \operatorname{Tr}\left(A_{\mu}^{I} J_{A_{I}}^{\mu}\right),
$$

where the mass matrix $M_{A}^{2}$ can be written in blocks as

$$
M_{A}^{2}=\left(\begin{array}{ccc}
M_{\ell \ell}^{2} & M_{\ell E}^{2} & M_{\ell N}^{2} \\
M_{E \ell}^{2} & M_{E E}^{2} & 0_{8 \times 3} \\
M_{N \ell}^{2} & 0_{3 \times 8} & M_{N N}^{2}
\end{array}\right)
$$




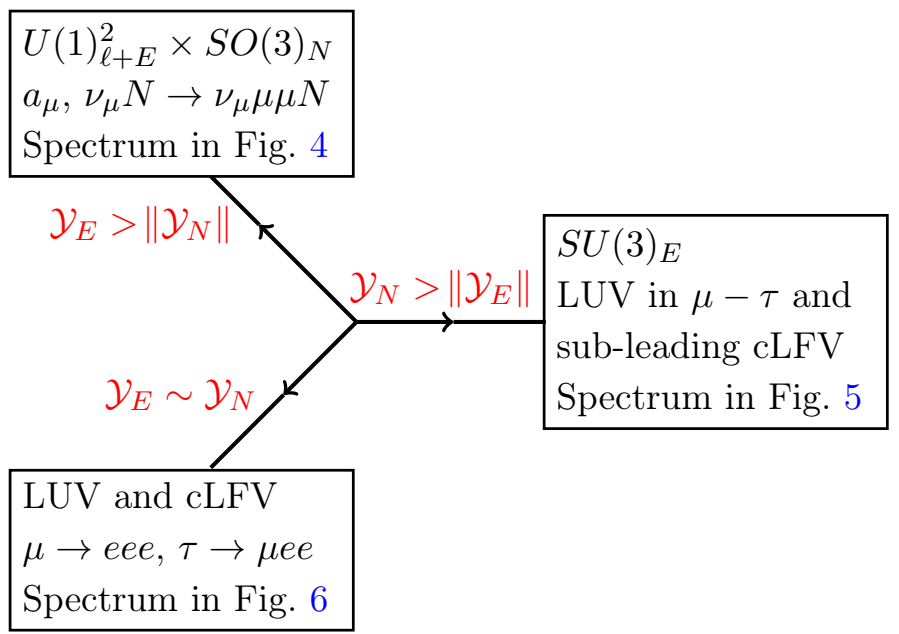

Figure 3. Schematic diagram for the relevant phenomenology scenarios. Each box reports the symmetry associated to the relevant gauge bosons and the expected dominant phenomenology.

with $\left(M_{E E}^{2}\right)_{i j}$ and $\left(M_{\ell E}^{2}\right)_{i j}=\left(M_{E \ell}^{2}\right)_{j i}$ identical to those in eq. (2.22) for the gauged-flavour SM case, while instead

$$
\begin{aligned}
\left(M_{\ell \ell}^{2}\right)_{i j} & =g_{\ell}^{2}\left\{\operatorname{Tr}\left(\mathcal{Y}_{E}\left\{T_{i}, T_{j}\right\} \mathcal{Y}_{E}^{\dagger}\right)+\operatorname{Tr}\left(\mathcal{Y}_{N}\left\{T_{i}, T_{j}\right\} \mathcal{Y}_{N}^{\dagger}\right)\right\} \\
\left(M_{\ell N}^{2}\right)_{i \hat{j}} & =\left(M_{N \ell}^{2}\right)_{\hat{j} i}=-2 g_{\ell} g_{N} \operatorname{Tr}\left(T_{i} \mathcal{Y}_{N}^{\dagger} T_{\hat{j}}^{\prime} \mathcal{Y}_{N}\right), \\
\left(M_{N N}^{2}\right)_{\hat{i} \hat{j}} & =g_{N}^{2} \operatorname{Tr}\left(\mathcal{Y}_{N}^{\dagger}\left\{T_{\hat{i}}^{\prime}, T_{\hat{j}}^{\prime}\right\} \mathcal{Y}_{N}\right)
\end{aligned}
$$

where $T^{\prime} \equiv\left\{T_{2}, T_{5}, T_{7}\right\}, i, j=\{1, \ldots, 8\}$ and $\hat{i}, \hat{j}=\{1, \ldots, 3\}$.

Notice that, contrary to the processes mediated by the exchange of $\mathrm{SU}(3)_{E}$ gauge bosons $A_{\mu}^{E}$, those mediated by $A_{\mu}^{\ell}$ can indeed lead to observable flavour non-conserving processes given the non-diagonal flavour structure of $\mathcal{Y}_{N}$ and the related low scales allowed in this gauged-flavour type I Seesaw scenario.

Generally speaking, $M_{A^{\ell}}$ will be determined by the largest value between $\left\|\mathcal{Y}_{E}\right\|$ and $\left\|\mathcal{Y}_{N}\right\|$. There are in general too many parameters to make definite predictions, though. The most relevant consequences are briefly discussed next and illustrated in figure 3 for three relevant limits: $\mathcal{Y}_{E}>\left\|\mathcal{Y}_{N}\right\|, \mathcal{Y}_{E} \sim \mathcal{Y}_{N}$ and $\left\|\mathcal{Y}_{E}\right\|<\mathcal{Y}_{N}$, with the latter two cases being of special phenomenological interest as they lead to putatively observable cLFV in addition to LUV signals.

\subsection{1 $\mathcal{Y}_{E}>\left\|\mathcal{Y}_{N}\right\|$ - vectorial flavour-preserving gauge bosons}

The heaviest gauge bosons would be those whose mass is dominated by the vev of $\mathcal{Y}_{E}$. This applies to all $\mathrm{SU}(3)_{\ell}$ and $\mathrm{SU}(3)_{E}$ gauge bosons but two (see below), as $\mathcal{Y}_{E}$ transforms under those two groups. The hierarchical structure of $\mathcal{Y}_{E}$ - with eigenvalues inversely proportional to the SM charged lepton masses - results in a stratification of those heavier gauge bosons in two layers, as illustrated by the two upper layers of the spectrum in figure 4: the upper level contains the nine gauge bosons which couple to the electron, while 


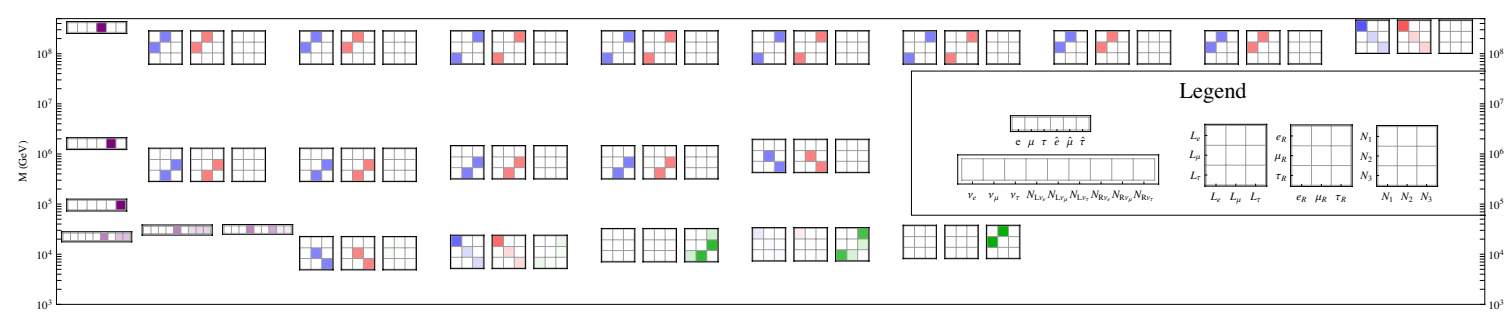

Figure 4. Gauge and fermion heavy spectrum for the gauged lepton flavour type I Seesaw model, with $\mathcal{Y}_{E} \gg\left\|\mathcal{Y}_{N}\right\|$. Boxes correspond to flavour gauge fields and lines to mirror fermions. Neutrino normal ordering was assumed and the parameter values taken are $\theta_{23}=45^{\circ}, \theta_{12}=33^{\circ}, \theta_{13}=8.8^{\circ}$, Dirac CP phase $\delta=3 \pi / 2$, Majorana phases $\alpha_{1}=\alpha_{2}=0, R=1$. All g's and all $\lambda^{\prime} s$ are 0.1 except $\lambda_{N}=1$ and $\mu_{E}=1 \mathrm{TeV}, \mu_{\mathrm{LN}}=1 \mathrm{KeV}$, while $m_{\nu_{1}}=0.03 \mathrm{eV}$.

the intermediate level corresponds to those gauge bosons coupling only to muons and taus. The phenomenological impact of the upper level will be neglected in what follows.

The lightest gauge bosons would be those which acquire instead a mass only through the vev of $\mathcal{Y}_{N}$. There are five such states. Three of them are the $\mathrm{SO}(3)_{N}$ gauge bosons, depicted (in green) in the illustrative case in figure 4: they carry flavour, mediating transitions only in the $N_{i}$ realm. Notice that they will only mix for complex $\mathcal{Y}_{N}$, since the mass cross-term that connects them to the other gauge bosons is $\operatorname{Tr}\left[T_{3,8} \mathcal{Y}_{N}^{\dagger} T_{2,5,7} \mathcal{Y}_{N}\right]=$ $-\operatorname{Tr}\left[T_{3,8} \mathcal{Y}_{N}^{T} T_{2,5,7} \mathcal{Y}_{N}^{*}\right]$, see eq. (3.19).

The presence of the other two light eigenstates - the lightest ones in figure 4 can be understood from the fact that $\mathcal{Y}_{E}$ can be made diagonal via a rotation in flavour space. This corresponds to the three distinct vectorial and diagonal U(1) symmetries which are preserved: LN which has not been gauged, plus two others which correspond to very light gauge bosons, which acquire a mass only through the vev of $\mathcal{Y}_{N}$. These states are diagonal in flavour space and traceless - see figure 4 - and given by the linear combination $A_{\mu}^{V}=\left(g_{E} A_{\mu}^{\ell}+g_{\ell} A_{\mu}^{E}\right) /\left(g_{\ell}^{2}+g_{E}^{2}\right)^{1 / 2}$, with mass matrix

$$
M_{A_{V}}^{2} \equiv 2 g_{\ell}^{2}\left(\begin{array}{ll}
\operatorname{Tr}\left(T_{3} \mathcal{Y}_{N}^{\dagger} \mathcal{Y}_{N} T_{3}\right) & \operatorname{Tr}\left(T_{3} \mathcal{Y}_{N}^{\dagger} \mathcal{Y}_{N} T_{8}\right) \\
\operatorname{Tr}\left(T_{8} \mathcal{Y}_{N}^{\dagger} \mathcal{Y}_{N} T_{3}\right) & \operatorname{Tr}\left(T_{8} \mathcal{Y}_{N}^{\dagger} \mathcal{Y}_{N} T_{8}\right)
\end{array}\right)
$$

Those two gauge bosons generically couple to all flavours with similar strength, see eq. (3.20), and thus the most stringent bound stems from LEP [53],

$$
M_{A_{V_{1}}} \geq 2.1 \times 10^{2} \mathrm{GeV}
$$

where $A_{V_{1}}$ denotes the lightest eigenstate of eq. (3.20). Those two vector bosons also contribute constructively ${ }^{11}$ to the muon anomalous magnetic moment:

$$
\delta a_{\mu}=\frac{m_{\mu}^{2}}{12 \pi^{2}} \times \frac{g_{E}^{2} g_{\ell}^{2}}{g_{\ell}^{2}+g_{E}^{2}} \sum_{i j} T_{i}^{\mu \mu}\left(M_{A_{V}}^{-2}\right)_{i j} T_{j}^{\mu \mu} .
$$

\footnotetext{
${ }^{11}$ As opposed to the contribution studied in eq. (2.49), in this case the sign is positive since the coupling of the lightest flavour gauge boson to leptons is vectorial.
} 
Although they could potentially explain the existing anomaly, this is excluded by neutrino trident production data, $\nu_{\mu} \mathcal{N} \rightarrow \nu_{\mu} \mu \mu \mathcal{N}$ with $\mathcal{N}$ denoting here a nucleus. Indeed, the contributions from the flavour gauge bosons to this observable read [54]

$$
\frac{\sigma^{(S M+A)}}{\sigma^{(S M)}}=\frac{1+\left(1+4 s_{W}^{2}+2 \delta_{V}\right)^{2}}{1+\left(1+4 s_{W}^{2}\right)^{2}}, \quad \delta_{V}=v^{2} \frac{g_{E}^{2} g_{\ell}^{2}}{g_{\ell}^{2}+g_{E}^{2}} \sum_{i j} T_{i}^{\mu \mu}\left(M_{A_{V}}^{-2}\right)_{i j} T_{j}^{\mu \mu}
$$

and are constrained by the CCFR [55] and CHARM-II [56] collaborations, implying the indirect bound $\delta a_{\mu}<7.5 \times 10^{-10}$, which precludes an explanation of the muon magnetic moment anomaly via these gauge bosons.

Figure 4 also illustrates that the lightest exotic neutral fermions would be those mirroring the light neutrino sector, as expected since the mirror fermion masses are linearly proportional to the flavon vevs. Therefore, the unitarity deviation $\Theta_{\nu}$ induced in the PMNS matrix by the mirror neutrinos dominates over $\Theta$ (stemming from the mirror charged leptons), see eq. (3.11). Analyses probing flavour non-conserving processes and electroweak precision data $[40,57-81]$ can then be translated into constrains on the combination $\Theta_{\nu} \Theta_{\nu}^{\dagger}[39]$ as follows:

$$
\begin{aligned}
& \left(\Theta_{\nu} \Theta_{\nu}^{\dagger}\right)_{e e}<2.5 \times 10^{-3}, \quad\left(\Theta_{\nu} \Theta_{\nu}^{\dagger}\right)_{e \mu}<2.4 \times 10^{-5}, \\
& \left(\Theta_{\nu} \Theta_{\nu}^{\dagger}\right)_{\mu \mu}<4.0 \times 10^{-4}, \quad\left(\Theta_{\nu} \Theta_{\nu}^{\dagger}\right)_{e \tau}<2.7 \times 10^{-3}, \\
& \left(\Theta_{\nu} \Theta_{\nu}^{\dagger}\right)_{\tau \tau}<5.6 \times 10^{-3}, \quad\left(\Theta_{\nu} \Theta_{\nu}^{\dagger}\right)_{\mu \tau}<1.2 \times 10^{-3} \text {, }
\end{aligned}
$$

at $95 \%$ CL.

\subsection{2 $\mathcal{Y}_{N}>\left\|\mathcal{Y}_{E}\right\|-$ LUV and subleading cLFV}

In this limit, in which all entries of $\mathcal{Y}_{N}$ are larger than the largest one in $\mathcal{Y}_{E}$, the lightest gauge bosons correspond to the $\mathrm{SU}(3)_{E}$ symmetry. Therefore, the leading phenomenology described in section 2 when gauging only the SM leptonic flavour group $\mathrm{SU}(3)_{\ell} \times \mathrm{SU}(3)_{E}$ will apply. In particular, as $\left\|\mathcal{Y}_{E}\right\|$ dominates, an effective low-energy $\mathrm{SU}(2)_{E}$ symmetry is at play and mediated by the three lightest gauge bosons, while transitions involving the electron flavour will be additionally suppressed by $\left(m_{e} / m_{\mu}\right)^{2}$ with respect to those in the $\mu-\tau$ sector. The lepton universality violation effects associated to the $\mu-\tau$ sector and dominated by fermionic $\hat{\tau}$ exchanges found in section 2 are also valid for this case.

As for the heavier states, since the leading contribution to the $\mathrm{SU}(3)_{\ell}$ gauge boson masses is given by $\mathcal{Y}_{N}$ no large hierarchies among the $\mathrm{SU}(3)_{\ell}$ gauge boson masses are expected for a generic $R$ matrix and generic light neutrino mass spectrum. Therefore, the importance of the lepton flavour violating processes mediated by these gauge bosons will not be strongly correlated to the specific flavours involved. This is in contrast to the case for $A_{\mu}^{E}$ shown in section 2.1. However, there are specific limiting cases with approximate symmetries for which hierarchies are introduced and the number of relevant parameters is reduced so that more definite predictions can be made. We briefly consider an example next. 


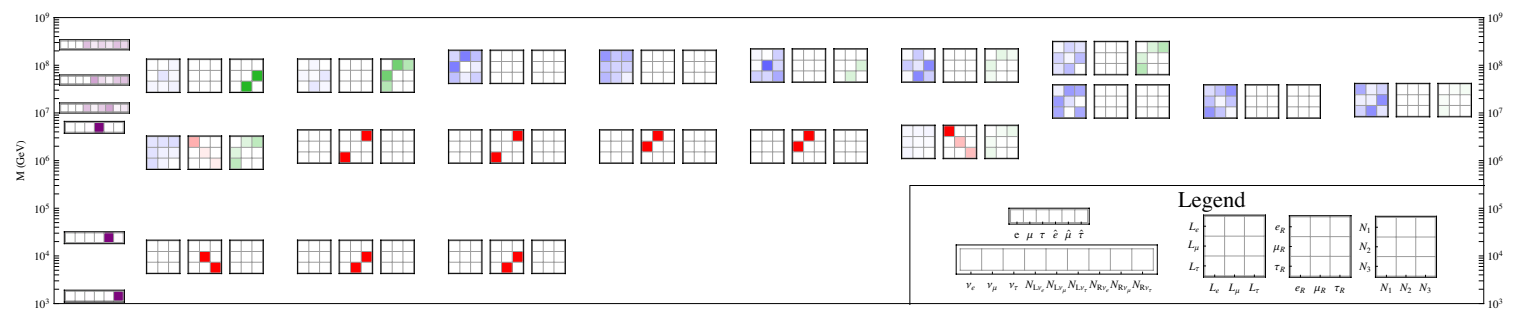

Figure 5. Gauge and fermion heavy spectrum for the gauged lepton flavour type I Seesaw model, with $\mathcal{Y}_{N}>\left\|\mathcal{Y}_{E}\right\|$ and degenerate light neutrinos, CP-odd case. Boxes correspond to flavour gauge fields and lines to mirror fermions. Neutrino normal ordering was assumed for neutrinos and the parameter values taken are $\theta_{23}=45^{\circ}, \theta_{12}=33^{\circ}, \theta_{13}=8.8^{\circ}$, Dirac CP phase $\delta=3 \pi / 2$, Majorana phases $\alpha_{21}=-\pi / 2, \alpha_{31}=-2 \pi / 3, R$ is a rotation in the 23 sector by angle $-i$ times a 12 rotation by angle $i$. All g's and all $\lambda^{\prime} s$ are 1 except $\lambda_{N}=2, \lambda_{\nu}=0.2, \mu_{E}=15 \mathrm{GeV}$, while $\mu_{\mathrm{LN}}=100 \mathrm{GeV}$ and $m_{\nu_{1}}=0.03 \mathrm{eV}$.

Generic $\boldsymbol{R}$ and degenerate neutrino masses. As expected, the lightest states of the spectrum will be similar to those discussed in section 2, as seen by comparing figure 2 and figure 5, while the heavier states can be now much lighter and thus of phenomenological interest, as explained earlier on.

In the limit of degenerate neutrinos, eqs. (3.6) and (3.8) lead to

$$
\mathcal{Y}_{N}=\frac{v}{\sqrt{2}} \frac{\lambda_{\nu} \sqrt{\mu_{\mathrm{LN}}}}{\lambda_{N} \sqrt{m_{\nu}}} R U^{\dagger} \equiv \frac{v}{\sqrt{2}} \frac{\lambda_{\nu} \sqrt{\mu_{\mathrm{LN}}}}{\lambda_{N} \sqrt{m_{\nu}}} e^{\eta_{i} T^{\prime i}} U^{\dagger} .
$$

This expression is invariant under a $\mathrm{U}(1)$ subgroup of $\mathrm{SU}(3)_{\ell} \times \mathrm{SO}(3)_{N}$ :

$$
\mathcal{Y}_{N} \rightarrow e^{i \alpha \eta_{i} T^{i}}\left(\mathcal{Y}_{N}\right) U e^{-i \alpha \eta_{i} T^{\prime i}} U^{\dagger},
$$

where $\alpha$ is the (real) parameter of the transformation. Therefore, the gauge boson associated with this $\mathrm{U}(1)$ will only acquire mass through $\mathcal{Y}_{E}$ and will be lighter than the rest. The generator of this residual $\mathrm{U}(1)$ symmetry in the $\mathrm{SU}(3)_{\ell}$ sector is $U \eta_{i} T^{\prime i} U^{\dagger}$ and therefore the induced cLFV four fermion operator mediated by that state is

$$
\frac{g_{\ell}^{2}}{M_{A_{\mathrm{U}(1)}}^{2}}\left(\bar{\ell}_{L} \gamma_{\mu} U \eta_{i} T^{\prime i} U^{\dagger} \ell_{L}\right)^{2} .
$$

That lighter state is illustrated by the first gauge boson on the second layer of figure 5 , in which generic values of the Dirac CP phase $\delta$ and a non-trivial $R$ matrix have been used. In this generic case, the most competitive bound on the operator in eq. (3.27) stems from the $\mu \rightarrow$ eee decay.

In the case of a $\mathrm{CP}$ conserving PMNS matrix, the antisymmetry of $T^{\prime i}$ would imply that the combination $U \eta_{i} T^{\prime i} U^{\dagger}$ in eq. (3.27) would have vanishing flavour diagonal interactions. The only expected decays would then be $\tau \rightarrow \mu e e$ and $\tau \rightarrow \mu \mu e$, determined by the specific values of $R$. Nevertheless, the recent hints $[82,83]$ of a leptonic CP phase $\delta \sim 270^{\circ}$ would discard this possibility, if confirmed. In this perspective, we refrain as well from detailing other specific predictions that would follow for scenarios with $\delta=0$ or $\pi$. 


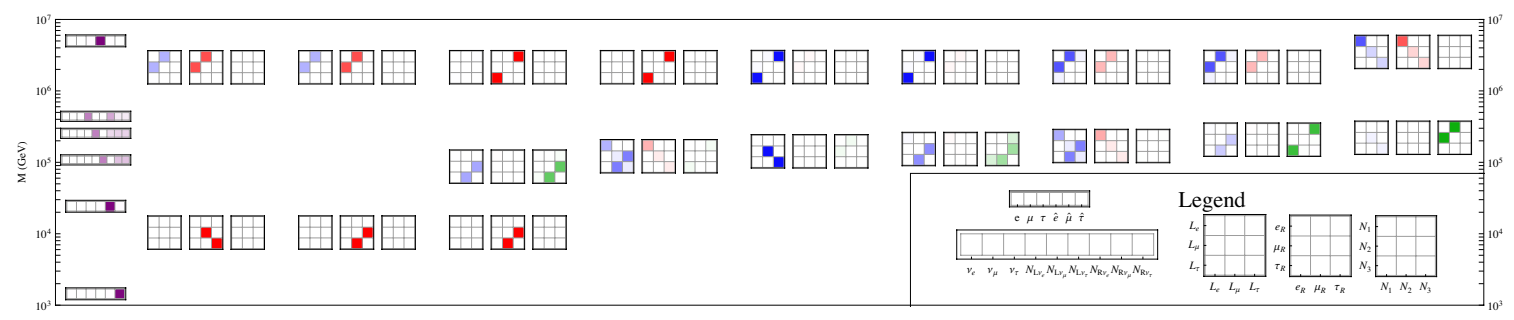

Figure 6. Gauge and fermion heavy spectrum for the gauged lepton flavour type I Seesaw model, with $\mathcal{Y}_{E} \sim\left\|\mathcal{Y}_{N}\right\|$. Boxes correspond to flavour gauge fields and lines to mirror fermions. Neutrino normal ordering was assumed and the parameter values taken are $\theta_{23}=45^{\circ}, \theta_{12}=33^{\circ}, \theta_{13}=8.8^{\circ}$, Dirac CP phase $\delta=3 \pi / 2$, Majorana phases $\alpha_{1}=\alpha_{2}=0, R=1$, all $\lambda$ 's and $g$ 's are taken to be 0.1 except $\lambda_{N}=1$ and $\mu_{E}=15 \mathrm{GeV}, \mu_{\mathrm{LN}}=20 \mathrm{KeV}$ and $m_{\nu_{1}}=0.003 \mathrm{eV}$.

\subsection{3 $\mathcal{Y}_{E} \sim\left\|\mathcal{Y}_{N}\right\|-$ LUV and cLFV}

This case is involved given the interplay of several scales, although it can be described qualitatively. As $\mathcal{Y}_{E}$ is intrinsically hierarchical (and determined by the inverse of the charged lepton masses), in the example considered next it is assumed that the norm $\left\|\mathcal{Y}_{N}\right\|$ is heavier than the eigenstates of the approximate $\mathrm{SU}(2)_{E}$ symmetry of the muon-tau sector and lighter than the rest of the $\mathcal{Y}_{E}$ entries. In consequence, the lightest exotic fermion and gauge boson masses are as in the SM gauged case discussed in section 2, as can be seen by comparing figure 2 with the illustrative case in figure 6 . The lightest fields in the spectrum are again the mirror $\hat{\tau}$ lepton and the $\mathrm{SU}(2)_{E}$ gauge bosons, leading to the $\mu-\tau$ phenomenology discussed in section 2 .

Additionally, the gauge bosons which take their masses dominantly from $\mathcal{Y}_{N}$ may now lead to observable cLFV signals, as discussed next. Electron number violation will be suppressed by the largest of the two scales $\left\|\mathcal{Y}_{E}\right\|$ and $\left\|\mathcal{Y}_{N}\right\|$, while muon and tau violation by the largest of $\left\|\mathcal{Y}_{E}\right\| m_{e} / m_{\mu}$ and $\left\|\mathcal{Y}_{N}\right\|$. Therefore, the generic expectations for flavour violating processes are:

$$
\begin{aligned}
& \operatorname{Br}_{\mu \rightarrow e e e}\left(A_{\mu}^{\ell}\right), \quad \operatorname{Br}_{\tau \rightarrow \mu e^{-} e^{-}}\left(A_{\mu}^{\ell}\right), \quad \operatorname{Br}_{\tau \rightarrow \mu \mu e}\left(A_{\mu}^{\ell}\right) \sim\left(\left\|\mathcal{Y}_{E}\right\|^{2}+\left\|\mathcal{Y}_{N}\right\|^{2}\right)^{-2}, \\
& \operatorname{Br}_{\tau \rightarrow \mu \mu \mu}\left(A_{\mu}^{\ell}\right), \quad \operatorname{Br}_{\tau \rightarrow \mu e^{+} e^{-}}\left(A_{\mu}^{\ell}\right) \sim\left(\frac{m_{e}^{2}}{m_{\mu}^{2}}\left\|\mathcal{Y}_{E}\right\|^{2}+\left\|\mathcal{Y}_{N}\right\|^{2}\right)^{-2} .
\end{aligned}
$$

The experimental bounds in table 3 can then be translated into limits on the combinations

$$
\begin{aligned}
\sqrt{\left\|\mathcal{Y}_{E}\right\|^{2}+\left\|\mathcal{Y}_{N}\right\|^{2}} & \geq 3.5 \times 10^{5} \mathrm{GeV}, \quad \text { from } \quad \mu \rightarrow \text { eee }, \\
\sqrt{\frac{m_{e}^{2}}{m_{\mu}^{2}}\left\|\mathcal{Y}_{E}\right\|^{2}+\left\|\mathcal{Y}_{N}\right\|^{2}} & \geq 1.9 \times 10^{4} \mathrm{GeV}, \quad \text { from } \tau \rightarrow \mu e^{+} e^{-} .
\end{aligned}
$$

When the two scales $\left\|\mathcal{Y}_{E}\right\|$ and $\left\|\mathcal{Y}_{N}\right\|$ are comparable, $\mu \rightarrow$ eee sets a lower bound on each of them of $\sim 2.5 \times 10^{5} \mathrm{GeV}$; when instead $\left\|\mathcal{Y}_{N}\right\|<\left\|\mathcal{Y}_{E}\right\|, \tau \rightarrow \mu e^{+} e^{-}$leads to a stronger bound on $\left\|\mathcal{Y}_{E}\right\| \gtrsim 2.9 \times 10^{6} \mathrm{GeV}$. In both cases, flavour observables turn out to be more sensitive to the scale of the flavour gauge bosons than present collider data, as 


\begin{tabular}{|l|l|}
\hline $\operatorname{Br}(\mu \rightarrow e \gamma) \leq 5.7 \times 10^{-13}$ & $\operatorname{Br}(\tau \rightarrow \mu \gamma) \leq 4.4 \times 10^{-8}$ \\
\hline $\operatorname{Br}(\tau \rightarrow e \gamma) \leq 3.3 \times 10^{-8}$ & $\operatorname{Br}(\mu \rightarrow e e e) \leq 1.0 \times 10^{-12}$ \\
\hline $\operatorname{Br}(\tau \rightarrow e e e) \leq 2.7 \times 10^{-8}$ & $\operatorname{Br}(\tau \rightarrow \mu \mu \mu) \leq 2.1 \times 10^{-8}$ \\
\hline $\operatorname{Br}\left(\tau \rightarrow \mu^{+} \mu^{-} e\right) \leq 2.7 \times 10^{-8}$ & $\operatorname{Br}\left(\tau \rightarrow \mu \mu^{-} e^{+}\right) \leq 1.7 \times 10^{-8}$ \\
\hline $\operatorname{Br}\left(\tau \rightarrow \mu e^{+} e^{-}\right) \leq 1.8 \times 10^{-8}$ & $\operatorname{Br}\left(\tau \rightarrow \mu^{+} e^{-} e\right) \leq 1.5 \times 10^{-8}$ \\
\hline
\end{tabular}

Table 3. 90\% CL limits on flavour violating decays of a charged lepton into three other charged leptons [24].

the bounds on $\left\|\mathcal{Y}_{E}\right\|$ are stronger than that extracted from direct searches in eq. (2.50), $\left\|\mathcal{Y}_{E}\right\| \geq 7.4 \times 10^{4} \mathrm{GeV}$.

\section{Comparison with minimal lepton flavour violation, for $\mathcal{Y}_{N} \gg \mathcal{Y}_{E}$}

We have gauged in the preceding sections the maximal non-abelian leptonic global flavour symmetry of the SM and of the type I Seesaw Lagrangian. In doing so, we were inspired by the phenomenological successes of the MFV ansatz in which the Yukawa couplings are treated as scalar spurions. A pertinent question is then whether the resulting low-energy phenomenology described above is compatible with that expected in the original formulation of Minimal Lepton Flavour Violation (MLFV) [26] and subsequent works [27-30].

The low-energy effective Lagrangian of our gauged-flavour models will, by construction, be formally invariant under the spurion analysis of MLFV; the question is whether the analytic dependence on the scalar fields matches that in MLFV. It is shown below that this is not always the case, due mainly to the presence of additional gauge bosons in the gauged-flavour Lagrangians.

For definiteness, we focus here on the specific limit $\mathcal{Y}_{N} \gg \mathcal{Y}_{E}$, which applies both to the gauged-flavour SM described in section 2 and to one scenario of the gauged-flavour type I Seesaw model, see section 3.1.2. Integrating out the flavour gauge bosons and the mirror fermion fields in eqs. (2.2)-(2.6), (3.1) and (3.2), and restricting the expansion to order $\mathcal{Y}^{-2}$ in flavon fields vevs $\left(\mathcal{Y}_{E}\right.$ and $\left.\mathcal{Y}_{N}\right)$, the low-energy Lagrangian reads ${ }^{12}$

$$
\begin{aligned}
\mathscr{L}^{\mathrm{eff}}= & \left(-\bar{\ell}_{L} H \frac{\lambda_{E} \mu_{E}}{\lambda_{\mathcal{E}} \mathcal{Y}_{E}} e_{R}-\ell_{L}^{T} \tilde{H} \frac{C_{\nu}}{\Lambda_{\mathrm{LN}}} \tilde{H}^{T} \ell_{L}+\text { h.c. }\right) \\
& +i \bar{e}_{R} \frac{1}{\lambda_{\mathcal{E}}^{2}} \frac{\mu_{E}^{2}}{\mathcal{Y}_{E} \mathcal{Y}_{E}^{\dagger}} \not D e_{R}+i \bar{\ell}_{L} H \frac{\lambda_{E}^{2}}{\lambda_{\mathcal{E}}^{2}} \frac{1}{\mathcal{Y}_{E}^{\dagger} \mathcal{Y}_{E}} \not D\left(H^{\dagger} \ell_{L}\right)+i \bar{\ell}_{L} \widetilde{H} \frac{\lambda_{\nu}^{2}}{\lambda_{N}^{2}} \frac{1}{\mathcal{Y}_{N}^{\dagger} \mathcal{Y}_{N}} \not D\left(\widetilde{H}^{\dagger} \ell_{L}\right) \\
& -\frac{c_{E}}{2} \operatorname{Tr}\left[\frac{1}{\mathcal{Y}_{E}^{\dagger} \mathcal{Y}_{E}}\right]\left(\bar{e}_{R} \gamma_{\mu} e_{R}\right)^{2}-\frac{1}{2} \operatorname{Tr}\left[\frac{1}{\mathcal{Y}_{N}^{\dagger} \mathcal{Y}_{N}}\right]\left(\bar{\ell}_{L} \gamma_{\mu} \ell_{L}\right)\left[c_{\ell}\left(\bar{\ell}_{L} \gamma_{\mu} \ell_{L}\right)+2 c_{\ell E}\left(\bar{e}_{R} \gamma_{\mu} e_{R}\right)\right],
\end{aligned}
$$

\footnotetext{
${ }^{12}$ Recall that we are working on the convention in which $\mu_{E}$ and all $\lambda_{i}$ coefficients are real; otherwise all $\lambda_{i}^{2}$ should be traded by $\left|\lambda_{i}\right|^{2}$.
} 


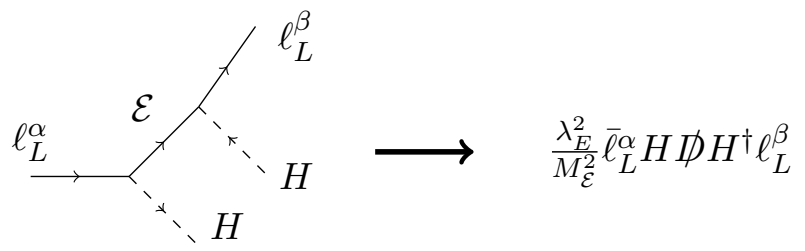

Figure 7. Example of effective operator induced via heavy fermion exchange.

where subleading contributions to the displayed operators have been neglected, e.g. $1 / \mathcal{Y}_{N}^{2}$ vs. $1 / \mathcal{Y}_{E}^{2}$, given that we assume $\mathcal{Y}_{N} \gg \mathcal{Y}_{E}$.

The first line in eq. (4.1) is in fact the general effective Lagrangian in eq. (1.4) which describes the charged lepton and neutrino masses, with the charged lepton Yukawa coupling given by $Y_{E}=\left(\lambda_{E} \mu_{E}\right) /\left(\lambda_{\mathcal{E}} \mathcal{Y}_{E}\right)$ in both gauged-flavour models considered, SM and type I Seesaw scenario, as already found in eq. (2.10) and section 3. $C_{\nu}$ is linear in $\mathcal{Y}_{N}^{-1}$ for the former scenario and quadratic for the latter, see respectively eqs. (2.10) and (3.5).

The second line in eq. (4.1) displays fermion-bilinear terms which are those resulting from integrating out the mirror fermions, as illustrated in figure 7. Finally, the last line stems from integrating out the heavy flavour gauge bosons resulting in effective four-fermion operators only; a flavour non-conserving operator resulting from $A_{\mu}^{\ell}$ exchange is depicted in figure 8 as illustration. The coefficient of the first four-fermion operator, $c_{E}$, has been given in eq. (2.45), whereas the explicit formulas for $c_{\ell}$ and $c_{\ell E}$ depend on the model under consideration; they will be discussed further below for phenomenologically accessible cases.

Mirror lepton exchange. The first term on the second line of eq. (4.1) contributes to the kinetic energy of the right-handed light charged leptons; the field redefinition

$$
e_{R} \rightarrow\left(1-\frac{1}{2 \lambda_{\mathcal{E}}^{2}} \frac{\mu_{E}^{2}}{\mathcal{Y}_{E}^{\dagger} \mathcal{Y}_{E}}\right) e_{R},
$$

allows to recover canonically normalized kinetic energies and leaves the rest of the Lagrangian unchanged, at the order considered. This confirms the result found in section 2, as the mixing $\Theta_{R}$ among right-handed charged fermions does not affect the gauge interactions.

The second term in that line is a dimension six $(d=6)$ effective operator with a coefficient of order $\mathcal{Y}_{E}^{-2}$ and therefore quadratic in the charged lepton Yukawa couplings $Y_{E}$, see eq. (2.10). Were one to write the $\mathcal{O}\left(Y_{E}^{2}\right)$ coefficient for such operator with the prescription of MLFV, it would read, in matrix notation,

$$
\text { MLFV: } \quad \frac{i}{\Lambda^{2}} \bar{\ell}_{L} H Y_{E} Y_{E}^{\dagger} \not D\left(H^{\dagger} \ell_{L}\right),
$$

which indeed corresponds to our result in eq. (4.1) provided the associated scale is identified as $\Lambda=\mu_{E}$, see eq. (2.10). Note that $\Lambda$ is then not the mass scale of any of the heavy particles in the model and can actually be lower. ${ }^{13}$

\footnotetext{
${ }^{13}$ If instead the coefficient is written in terms of mass scales, e.g. the mass of the lightest mirror charged lepton, $M_{\tau}$, it would read $\lambda_{E}^{2} / M_{\tau}^{2} \times Y_{E} Y_{E}^{\dagger} /\left\|Y_{E}^{2}\right\|$ to order $m_{\mu} / m_{\tau}$.
} 


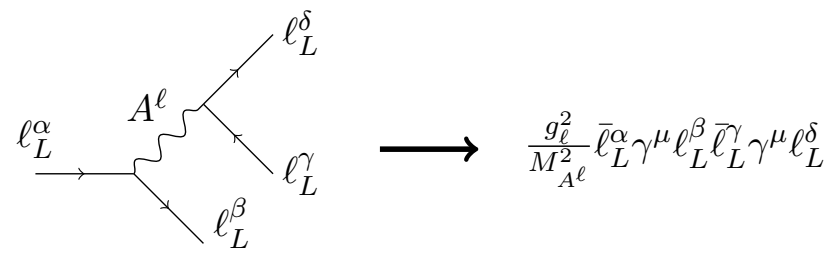

Figure 8. Tree-level exchange of a flavour gauge boson resulting in a four-fermion effective operator.

The rest of operators produced by fermion exchange can be cast as well in standard MLFV form; in particular the third operator in the second line of eq. (4.1) induces charged flavour violation as was indeed already studied in the context of leptonic MFV in ref. [29].

A relevant difference between MLFV constructions and the flavour-gauged scenario concerns $\mathrm{CP}$ violation. While a priori no symmetry principle prevents from assuming a complex overall phase in non-hermitian MLFV operators, in the lepton gauged-flavour models studied here such extra overall phases are absent. Therefore, the gauging of the lepton flavour symmetries provides a mechanism to protect against $\mathrm{CP}$ violation, not present in generic MLFV scenarios. In other words, the only source of CP violation are the scalar vevs and thus the only physical CP-odd phases are those of the PMNS matrix in both gauged-flavour scenarios, plus the usual extra phases of the minimal type I Seesaw model in the gauged-flavour type I Seesaw case.

Flavoured gauge boson exchange. The effective couplings resulting from the exchange of a heavy flavour gauge boson present a more complicated structure than those mediated by heavy fermions. For instance, the first operator in the third line of eq. (4.1) involves four right-handed charged lepton fields and a coefficient of order $\mathcal{Y}_{E}^{-2}$. Using eq. (2.45) and eq. (2.10), the dependence on the charged lepton Yukawa coupling $Y_{E}$ in the gauged-flavour case reads, in matrix notation,

$$
-\frac{1}{2} \sum_{k}(-1)^{k} \bar{e}_{R} \frac{\gamma_{\mu}}{\left(Y_{E}^{\dagger} Y_{E}\right)^{k}} e_{R} \bar{e}_{R} \gamma_{\mu}\left(Y_{E}^{\dagger} Y_{E}\right)^{k+1} e_{R}+\frac{1}{4 \operatorname{Tr}\left[Y_{E}^{\dagger} Y_{E}\right]}\left(\bar{e}_{R} \gamma_{\mu} Y_{E}^{\dagger} Y_{E} e_{R}\right)^{2},
$$

where $1 /(1+x)=\sum(-x)^{n}$ has been used. In contrast, within the MLFV prescription the Lagrangian term would be given by

$$
\mathrm{MFV}: \quad \frac{1}{\Lambda^{2}}\left(\bar{e}_{R} \gamma_{\mu} Y_{E}^{\dagger} Y_{E} e_{R}\right)\left(\bar{e}_{R} \gamma_{\mu} e_{R}\right),
$$

at leading order. In consequence, the spurion dependences do not match even if formally both are of order $Y_{E}^{2}$. Furthermore, only two leptons are involved in a non-trivial flavour structure in the MLFV case instead of four in the gauged-flavour scenario. In both cases, although this operator induces LUV, it does not induce LFV which is the distinctive feature of MLFV to which we now turn.

The second term in the third line of the Lagrangian eq. (4.1) exhibits a combination of two operators which induce LFV transitions - weighted down by $\mathcal{Y}_{N}^{-2}$ — which can be 
compared to the operators $O_{4 L}^{(1)}, O_{4 L}^{(2)}, O_{4 L}^{(3)}$ of ref. [27]. Those two operators are strongly suppressed in the gauged-flavour SM case as the $\mathcal{Y}_{N}$ scale is necessarily very high, while they may lead to visible effects in the context of the gauged-flavour type I Seesaw model in section 3.1.2, as the scale associated to $\mathcal{Y}_{N}$ can be low enough even if $\mathcal{Y}_{N}>\left\|\mathcal{Y}_{E}\right\|$. In the following, to allow a fair comparison with MLFV we will focus on flavour non-conserving transitions and consider a CP-even limit of the gauged-flavour type I Seesaw model.

CP invariance $\left(R=\mathbf{1}, \delta=\mathbf{0}, \alpha_{\mathbf{2 1}}=\alpha_{\mathbf{3 1}}=\mathbf{0}\right)$. In the CP-even limit considered, the combination of two operators appearing in the last term in eq. (4.1),

$$
-\frac{1}{2} \operatorname{Tr}\left[\frac{1}{\mathcal{Y}_{N}^{\dagger} \mathcal{Y}_{N}}\right]\left(\bar{\ell}_{L}^{\alpha} \gamma_{\mu} \ell_{L}^{\beta}\right)\left[c_{\ell}^{\alpha \beta \kappa \rho}\left(\bar{\ell}_{L}^{\kappa} \gamma^{\mu} \ell_{L}^{\rho}\right)+2 c_{\ell E}^{\alpha \beta \kappa \rho}\left(\bar{e}_{R}^{\kappa} \gamma^{\mu} e_{R}^{\rho}\right)\right],
$$

is determined by the coefficients given by

$$
c_{\ell}^{\alpha \beta \kappa \rho}=U_{\alpha i}^{\dagger} U_{j \beta} U_{\kappa r}^{\dagger} U_{s \rho} c_{\ell}^{i j r s}, \quad c_{\ell E}^{\alpha \beta \kappa \rho}=U^{\alpha i \dagger} U^{j \beta} c_{\ell}^{i j \kappa \rho},
$$

with

$$
\begin{aligned}
c_{\ell}^{i j r s}= & \frac{1}{\sum_{k} m_{\nu_{k}}}\left(\frac{\delta_{i s} \delta_{j r} m_{\nu_{i}} m_{\nu_{r}}\left(m_{\nu_{i}}^{2}+m_{\nu_{r}}^{2}\right)}{\left(m_{\nu_{i}}^{2}-m_{\nu_{r}}^{2}\right)\left(m_{\nu_{i}}-m_{\nu_{r}}\right)+\delta_{i r}\left(2 m_{\nu_{i}}\right)^{3}}\right. \\
& \left.-\frac{2 \delta_{i r} \delta_{j s} m_{\nu_{i}}^{2} m_{\nu_{j}}^{2}}{\left(m_{\nu_{i}}^{2}-m_{\nu_{j}}^{2}\right)\left(m_{\nu_{i}}-m_{\nu_{j}}\right)-\delta_{i j}\left(2 m_{\nu_{i}}\right)^{3}}-\frac{\delta_{i j} \delta_{r s} m_{\nu_{i}} m_{\nu_{r}}}{2 \sum_{k} m_{\nu_{k}}}\right) \\
c_{\ell E}^{i j \kappa \rho}= & \frac{m_{\kappa} m_{\rho}}{m_{\kappa}^{2}+m_{\rho}^{2}} \frac{1}{\sum_{k} m_{\nu_{k}}}\left(\frac{2 U_{\kappa j} U_{i \rho}^{\dagger} m_{\nu_{i}} m_{\nu_{j}}\left(m_{\nu_{i}}^{2}+m_{\nu_{j}}^{2}\right)}{\left(m_{\nu_{i}}^{2}-m_{\nu_{j}}^{2}\right)\left(m_{\nu_{i}}-m_{\nu_{j}}\right)+\delta_{i j}\left(2 m_{\nu_{i}}\right)^{3}}\right. \\
& \left.-\frac{4 U_{\kappa i} U_{j \rho}^{\dagger} m_{\nu_{i}}^{2} m_{\nu_{j}}^{2}}{\left(m_{\nu_{i}}^{2}-m_{\nu_{j}}^{2}\right)\left(m_{\nu_{i}}-m_{\nu_{j}}\right)-\delta_{i j}\left(2 m_{\nu_{i}}\right)^{3}}-\frac{\sum_{k} U_{\kappa \gamma} m_{\nu_{k}} U_{\gamma \rho}^{\dagger} \delta_{i j} m_{\nu_{i}}}{\sum_{k} m_{\nu_{k}}}\right),
\end{aligned}
$$

where the $c_{\ell}$ coefficients correspond to transitions between purely left-handed leptons, while $c_{\ell E}$ correspond to left-right mixed terms. ${ }^{14}$ Alike to the comparison between the operators in eqs. (4.4) and (4.5), the Yukawa dependence of the gauged-flavour model cannot be matched in this case to that in standard approaches to MLFV [26, 27]; we will compare here for definiteness with the "extended" model in ref. [26] for which the MLFV ansatz would suggest a coupling proportional to ${ }^{15}$

$$
\bar{\ell}_{L} \gamma_{\mu} U m_{\nu}^{\operatorname{diag}} U^{\dagger} \ell_{L} \bar{\ell}_{L} \gamma^{\mu} \ell_{L}
$$

The differences in the operator coefficients in eqs. (4.6)-(4.9) versus eq. (4.10) translate into distinctive phenomenological signals; as an illustration, the branching ratios for various

\footnotetext{
${ }^{14}$ The coefficients $c_{\ell E}^{i j \gamma \delta}$ appear suppressed with respect to $c_{\ell}^{i j k l}$ by a factor $m_{\gamma} m_{\delta} /\left(m_{\gamma}^{2}+m_{\delta}^{2}\right)$. This implies that left-right $c_{\ell E}$ contributions to transitions between leptons of neighbouring flavours (e.g. $\mu \rightarrow e e e$ and $\tau \rightarrow \mu \mu \mu$ ) are larger than between the third to the first generations (e.g., $\tau \rightarrow$ eee or $\tau \rightarrow \mu e e$ ).

${ }^{15}$ In the notation of our gauged-flavour type I Seesaw model in section 3 , the coefficient in front of this equation would read $\left(v^{2} \mu_{\mathrm{LN}}\right)^{-1}$, see footnote 9 .
} 


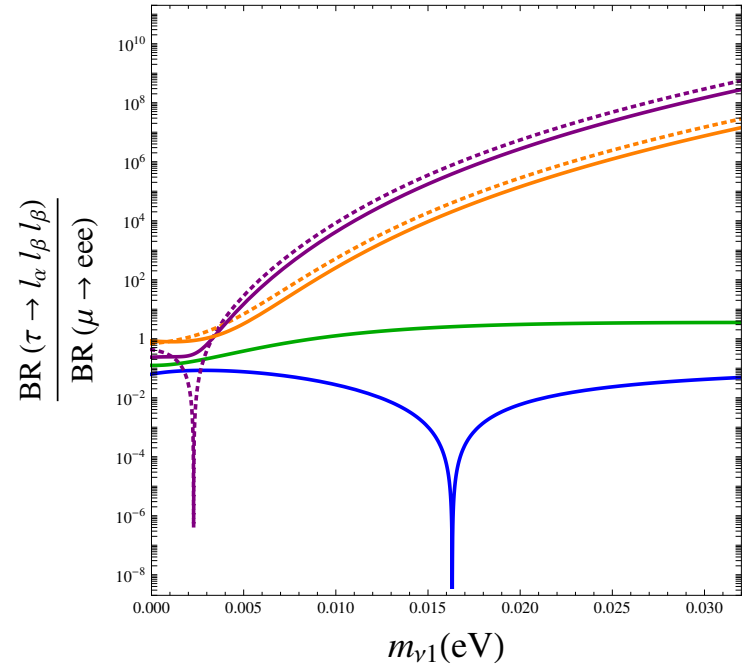

(a) Gauged Flavour, NO.

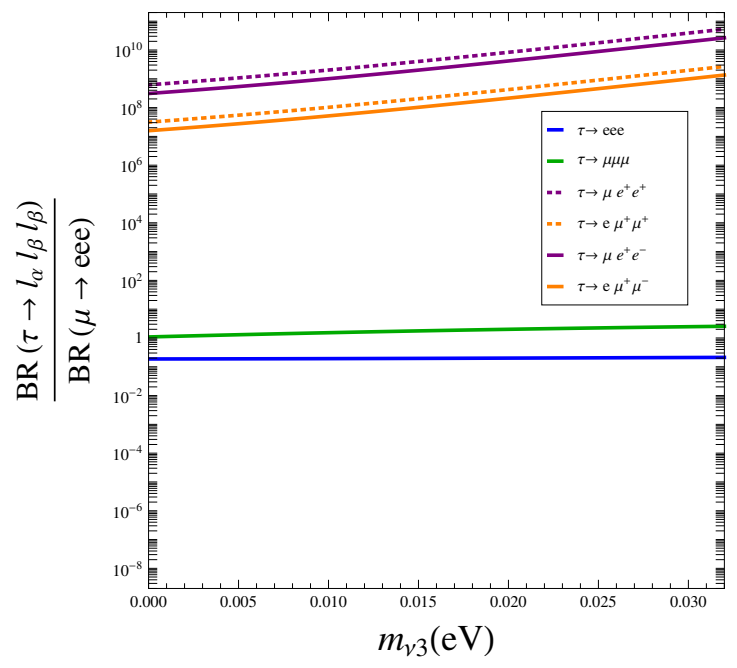

(c) Gauged Flavour, IO.

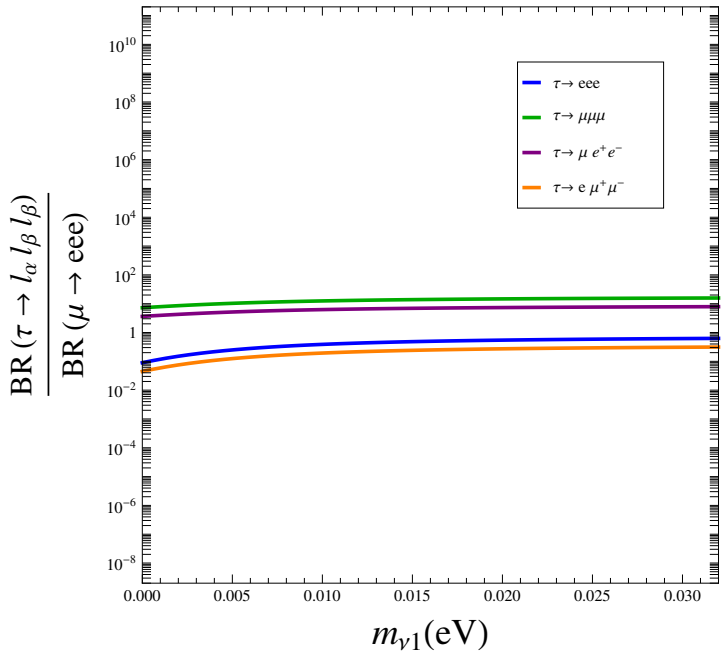

(b) MLFV, NO.

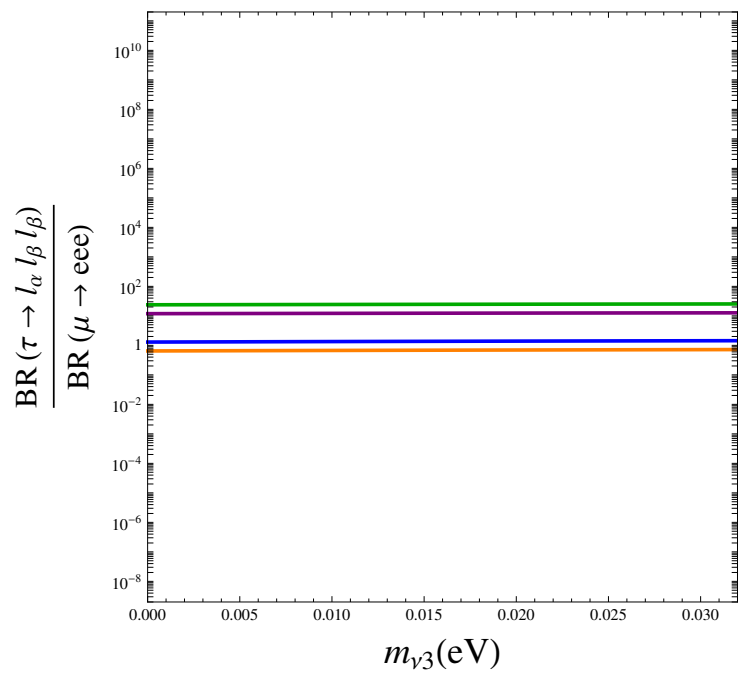

(d) MLFV, IO.

Figure 9. Comparison between the gauged-flavour type-I Seesaw scenario and MLFV in a CP-even case: branching ratios for the different lepton rare decays over that for $\mu \rightarrow e e e$, for neutrino normal ordering (NO) and inverted ordering (IO).

$l_{\alpha} \rightarrow l_{\beta} l_{\rho}^{+} l_{\kappa}^{-}$processes are compared in figure 9. A first clear difference is the absence of processes that violate lepton flavour by two units in the MLFV case, e.g., $\tau \rightarrow \mu e^{+} e^{+}$and $\tau \rightarrow e \mu^{+} \mu^{+}$(the dashed lines in the gauged-flavour case). These processes are suppressed in MLFV by higher-order spurion insertions, while the more intricate dependence on Yukawa couplings of the gauged-flavour case allows them at leading order.

A second prominent feature depicted in figure 9 is the strong hierarchy between two different type of decays in the gauged-flavour scenario, for inverted neutrino hierarchy 
and also for normal ordering with large $m_{\nu_{1}}$ : transitions involving only one flavour in the final state are much suppressed, see figures $9 \mathrm{a}$ and $9 \mathrm{c}$, unlike in MLFV, figures $9 \mathrm{~b}$ and 9d. In consequence, the dominant channels for the gauged-flavour scenario are $\tau \rightarrow \mu e e$ and $\tau \rightarrow e \mu \mu$ (in purple and orange). This hierarchy can be understood in terms of symmetry. If the three light neutrinos are almost degenerate, an approximate $\mathrm{SO}(3)_{\ell+N}$ remains unbroken, as already pointed out in refs. [13, 14]. The three corresponding gauge bosons would therefore be lighter than the rest with masses proportional to the neutrino mass splittings and thus suppressed by a factor $\left(m_{\nu_{i}}-m_{\nu_{j}}\right) /\left(m_{\nu_{i}}+m_{\nu_{j}}\right)$. The lightest of these gauge bosons corresponds to the smallest mass splitting $\left(\Delta m_{\text {sol }}^{2} \approx 7.50 \times 10^{-5} \mathrm{eV}^{2}\right)$ between $m_{\nu_{2}}$ and $m_{\nu_{1}}$, and dominates the contribution for inverted neutrino hierarchy as well as for normal ordering with large $m_{\nu_{1}}$. Because the couplings of this lightest flavour gauge boson are given by the generator of $\mathrm{SO}(2)$ rotations, which is antisymmetric in flavour, a selection rule for the decays follows. This can be seen explicitly in the limit $\Delta m_{\text {sol }} \ll \sum m_{\nu_{i}}$ in which eqs. (4.6)-(4.8) simplify to

$$
\simeq-\frac{\left\|\mathcal{Y}_{N}^{-1}\right\|^{2}}{54} \frac{\left(\sum_{k} m_{\nu_{k}}\right)^{2}}{\Delta m_{\mathrm{sol}}^{2}}\left(U_{\alpha 1} U_{2 \beta}^{\dagger}-U_{\alpha 2} U_{1 \beta}^{\dagger}\right)\left(U_{\gamma 1} U_{2 \delta}^{\dagger}-U_{\gamma 2} U_{1 \delta}^{\dagger}\right) \bar{\ell}_{L}^{\alpha} \gamma_{\mu} \ell_{L}^{\beta} \bar{\ell}_{L}^{\gamma} \gamma^{\mu} \ell_{L}^{\delta},
$$

from which it follows that whenever two flavours coincide, given the assumption of CP invariance the corresponding operator coefficient vanishes an hence $l \rightarrow l^{\prime} l^{\prime} l^{\prime}$ cancels, whereas for more than two flavours involved

$$
\frac{\operatorname{Br}(\tau \rightarrow \mu e e)}{\operatorname{Br}(\tau \rightarrow \mu \mu e)}=\frac{\sin ^{2}\left(\theta_{23}\right)}{\sin ^{2}\left(\theta_{13}\right)} \sim 20 .
$$

In contrast, in MLFV the $\tau \rightarrow \mu \mu \mu$ and $\tau \rightarrow$ eee branching ratios are a factor two - due to combinatorics - times those for $\tau \rightarrow \mu e^{+} e^{-}$and $\tau \rightarrow e \mu^{+} \mu^{-}$, respectively, see figures $9 \mathrm{~b}$ and $9 \mathrm{~d}$.

\section{Conclusions}

We have considered the gauging of leptonic global flavour symmetries that the SM Lagrangian or its fermionic Seesaw extension exhibit in the limit of negligible light lepton masses. A remarkable consequence is that the gauge anomaly cancellation conditions point to a universal underlying Seesaw pattern for both charged and neutral leptons:

- The gauging of the flavour symmetry $\mathrm{SU}(3)_{\ell} \times \mathrm{SU}(3)_{E}$ of the SM Lagrangian (that is, without assuming right-handed neutrinos) leads to the minimal type I Seesaw scenario as the simplest realization in terms of extra fields needed. In other words, without assuming Majorana neutrino masses, the gauging procedure suggests them directly.

- Starting instead from the maximal flavour symmetry of the type I Seesaw Lagrangian, $\mathrm{SU}(3)_{\ell} \times \mathrm{SU}(3)_{E} \times \mathrm{SO}(3)_{N}$, leads to a double Seesaw and in particular an inverse Seesaw pattern. 
This study extends previous work on gauging the flavour symmetries of the SM quark sector, which had already shown the existence of a Seesaw-like pattern that protected the model from the customary FCNC issues which tend to be the graveyard of attempts to understand dynamically the flavour puzzle. Interesting signals and correlations have been identified here as a result of gauging the maximal non-abelian flavour symmetries of the SM and of the type I Seesaw Lagrangian. The main leptonic flavour signals expected tend to involve the heavier SM leptons, whose interactions are less constrained by present data.

In the leptonic gauged-flavour SM case, the expected phenomenological signals are flavour-conserving, and include charged-lepton universality violation and non-unitarity of the PMNS matrix that follow from the (flavour diagonal) modifications of the couplings of leptons to $Z$ and $W$ bosons, particularly prominent for $\tau$-related observables. Furthermore, the first particles awaiting discovery would be a tau mirror lepton and $\mathrm{SU}(3)_{E}$ gauge bosons which mediate $\mu_{R}-\tau_{R}$ transitions.

Gauging instead the maximal lepton flavour symmetry of type I Seesaw may lead not only to signals of lepton universality violation but also to putatively observable flavour non-conserving transitions among charged leptons. The dominant signals expected depend mainly on the relative hierarchy of the scalar vevs that generate the charged lepton masses $\left\|\mathcal{Y}_{E}\right\|$ versus those that generate the neutrino ones $\left\|\mathcal{Y}_{N}\right\|$ and the LN scale. When all $\mathcal{Y}_{E}$ vevs are larger than $\left\|\mathcal{Y}_{N}\right\|$, the leading transitions are again flavour-conserving, while the lightest states in the spectrum are mirror neutrinos and gauge bosons whose mass is determined by $\left\|\mathcal{Y}_{N}\right\|$. In the opposite case, that is for $\left\|\mathcal{Y}_{N}\right\|>\left\|\mathcal{Y}_{E}\right\|$, the lowest states are again the mirror tau lepton and the three $\mathrm{SU}(3)_{E}$ gauge bosons which mediate transitions in the $\mu_{R}-\tau_{R}$ sector. Of particular interest is the fact that Majorana masses within an approximate $\mathrm{U}(1)$ lepton number symmetry setup are allowed, associated to the inverse Seesaw structure that results naturally from the requirement of gauge anomaly cancellation; it is precisely because the lepton scale is then distinct from the lepton number scale, that the latter can be low enough to expect sizeable flavour-changing signals. The precise phenomenology depends much on the $\mathrm{CP}$ pattern of the model. For the generic case of $\mathrm{CP}$ violation and (almost degenerate) neutrinos, $\mu \rightarrow$ eee is at present the most sensitive flavour non-conserving channel.

The results have been also compared with the phenomenological predictions of leptonic minimal flavour violation. We have shown that the presence of additional flavour gauge bosons may provide distinct low-energy transitions among the SM fields. It is also remarkable that the gauging of the lepton flavour symmetries provides a mechanism to protect against extra sources of CP violation beyond those in the SM (and Seesaw type I), which is absent in generic minimal lepton flavour violation scenarios. In addition, flavour changing transitions among charged leptons involving more than two distinct leptons tend to be stronger than those in which a tau or muon decays into three equal leptons, in contrast again with generic minimal flavour violation. The impact of scalar flavour excitations is model-dependent and remains to be studied in detail, although it is expected to abide by the same flavour protection than the rest of the theory.

The necessary mediation of at least one BSM field is at the basis of the Seesaw mechanism for the generation of light neutrino Majorana masses; it is very suggestive that the 
mass mechanism for light fermions - quarks and leptons - which results from gauging the flavour symmetries corresponds qualitatively to the same pattern. Interestingly, other theoretical constructions such as "partial compositeness" lead as well to a universal Seesaw-like pattern behind fermion masses; if new flavour signals are indeed observed, an extended and detailed study of many flavour channels will be needed to disentangle a possible flavoured-gauge origin. The main drawback of our construction is our ignorance about the absolute value of the scales involved, that could render the predictions of these models out of reach in the foreseeable future. Yet, the quest to identify a dynamical origin to the flavour puzzle is a fundamental and fascinating endeavour plausibly awaiting discovery.

\section{Acknowledgments}

We thank specially Gino Isidori and Luciano Maiani for initial discussions. We are also indebted to Andy Cohen, Paride Paradisi and Sara Saa for very useful comments. The work of RA and BG was supported in part by DOE grant DE-SC0009919. EFM, MBG, LM and PQ acknowledge partial financial support by the European Union through the FP7 ITN INVISIBLES (PITN-GA-2011-289442), by the Horizon2020-MSCA-RISE-2015//690575INVISIBLESPLUS, by the Horizon2020-MSCA-ITN-2015//674896-ELUSIVES, by CiCYT through the project FPA2012-31880, and by the Spanish MINECO through the Centro de excelencia Severo Ochoa Program under grant SEV-2012-0249. EFM also acknowledges support from the EU FP7 Marie Curie Actions CIG NeuProbes (PCIG11-GA-2012-321582) and the Spanish MINECO through the "Ramón y Cajal" programme (RYC2011-07710). The work of P.Q. is funded by Fundacion La Caixa under "La Caixa-Severo Ochoa" international predoctoral grant.

Open Access. This article is distributed under the terms of the Creative Commons Attribution License (CC-BY 4.0), which permits any use, distribution and reproduction in any medium, provided the original author(s) and source are credited.

\section{References}

[1] C.D. Froggatt and H.B. Nielsen, Hierarchy of Quark Masses, Cabibbo Angles and CP-violation, Nucl. Phys. B 147 (1979) 277 [inSPIRE].

[2] G. Isidori, Y. Nir and G. Perez, Flavor Physics Constraints for Physics Beyond the Standard Model, Ann. Rev. Nucl. Part. Sci. 60 (2010) 355 [arXiv:1002.0900] [inSPIRE].

[3] R.S. Chivukula and H. Georgi, Composite Technicolor Standard Model, Phys. Lett. B 188 (1987) 99 [INSPIRE].

[4] G. D'Ambrosio, G.F. Giudice, G. Isidori and A. Strumia, Minimal flavor violation: An Effective field theory approach, Nucl. Phys. B 645 (2002) 155 [hep-ph/0207036] [INSPIRE].

[5] A. Anselm and Z. Berezhiani, Weak mixing angles as dynamical degrees of freedom, Nucl. Phys. B 484 (1997) 97 [hep-ph/9605400] [InSPIRE].

[6] R. Barbieri, L.J. Hall, G.L. Kane and G.G. Ross, Nearly degenerate neutrinos and broken flavor symmetry, hep-ph/9901228 [INSPIRE]. 
[7] Z. Berezhiani and A. Rossi, Flavor structure, flavor symmetry and supersymmetry, Nucl. Phys. Proc. Suppl. 101 (2001) 410 [hep-ph/0107054] [INSPIRE].

[8] P.F. Harrison and W.G. Scott, Covariant extremisation of flavor-symmetric Jarlskog invariants and the neutrino mixing matrix, Phys. Lett. B 628 (2005) 93 [hep-ph/0508012] [INSPIRE].

[9] T. Feldmann, M. Jung and T. Mannel, Sequential Flavour Symmetry Breaking, Phys. Rev. D 80 (2009) 033003 [arXiv:0906.1523] [INSPIRE].

[10] R. Alonso, M.B. Gavela, L. Merlo and S. Rigolin, On the scalar potential of minimal flavour violation, JHEP 07 (2011) 012 [arXiv:1103.2915] [INSPIRE].

[11] R. Alonso, M.B. Gavela, D. Hernández and L. Merlo, On the Potential of Leptonic Minimal Flavour Violation, Phys. Lett. B 715 (2012) 194 [arXiv:1206.3167] [InSPIRE].

[12] J.R. Espinosa, C.S. Fong and E. Nardi, Yukawa hierarchies from spontaneous breaking of the $\mathrm{SU}(3)_{L} \times \mathrm{SU}(3)_{R}$ flavour symmetry?, JHEP 02 (2013) 137 [arXiv: 1211.6428] [INSPIRE].

[13] R. Alonso, M.B. Gavela, D. Hernández, L. Merlo and S. Rigolin, Leptonic Dynamical Yukawa Couplings, JHEP 08 (2013) 069 [arXiv:1306.5922] [INSPIRE].

[14] R. Alonso, M.B. Gavela, G. Isidori and L. Maiani, Neutrino Mixing and Masses from a Minimum Principle, JHEP 11 (2013) 187 [arXiv:1306.5927] [INSPIRE].

[15] B. Grinstein, M. Redi and G. Villadoro, Low Scale Flavor Gauge Symmetries, JHEP 11 (2010) 067 [arXiv: 1009.2049] [INSPIRE].

[16] Z.G. Berezhiani and M.Y. Khlopov, The Theory of broken gauge symmetry of families (in Russian), Sov. J. Nucl. Phys. 51 (1990) 739 [Yad. Fiz. 51 (1990) 1157] [InSPIRE].

[17] T. Feldmann, See-Saw Masses for Quarks and Leptons in SU(5), JHEP 04 (2011) 043 [arXiv: 1010.2116] [INSPIRE].

[18] D. Guadagnoli, R.N. Mohapatra and I. Sung, Gauged Flavor Group with Left-Right Symmetry, JHEP 04 (2011) 093 [arXiv: 1103.4170] [INSPIRE].

[19] A.J. Buras, L. Merlo and E. Stamou, The Impact of Flavour Changing Neutral Gauge Bosons on $\bar{B} \rightarrow X_{S} \gamma$, JHEP 08 (2011) 124 [arXiv: 1105.5146] [InSPIRE].

[20] A.J. Buras, M.V. Carlucci, L. Merlo and E. Stamou, Phenomenology of a Gauged SU $(3)^{3}$ Flavour Model, JHEP 03 (2012) 088 [arXiv: 1112.4477] [INSPIRE].

[21] B. Fornal, Baryon number violation beyond the standard model, Ph.D. Thesis, California Institute of Technology (Caltech) — Division of Physics, Mathematics and Astronomy (PMA), Pasadena California U.S.A. (2014) [inSPIRE].

[22] T. Feldmann, C. Luhn and P. Moch, Lepton-flavour violation in a Pati-Salam model with gauged flavour symmetry, JHEP 11 (2016) 078 [arXiv:1608.04124] [INSPIRE].

[23] F.S. Queiroz and W. Shepherd, New Physics Contributions to the Muon Anomalous Magnetic Moment: A Numerical Code, Phys. Rev. D 89 (2014) 095024 [arXiv:1403.2309] [INSPIRE].

[24] Particle Data Group collaboration, K.A. Olive et al., Review of Particle Physics, Chin. Phys. C 38 (2014) 090001 [inSPIRE].

[25] M.C. Gonzalez-Garcia, M. Maltoni and T. Schwetz, Updated fit to three neutrino mixing: status of leptonic CP-violation, JHEP 11 (2014) 052 [arXiv: 1409.5439] [INSPIRE]. 
[26] V. Cirigliano, B. Grinstein, G. Isidori and M.B. Wise, Minimal flavor violation in the lepton sector, Nucl. Phys. B 728 (2005) 121 [hep-ph/0507001] [INSPIRE].

[27] V. Cirigliano and B. Grinstein, Phenomenology of minimal lepton flavor violation, Nucl. Phys. B 752 (2006) 18 [hep-ph/0601111] [INSPIRE].

[28] S. Davidson and F. Palorini, Various definitions of Minimal Flavour Violation for Leptons, Phys. Lett. B 642 (2006) 72 [hep-ph/0607329] [INSPIRE].

[29] M.B. Gavela, T. Hambye, D. Hernández and P. Hernández, Minimal Flavour Seesaw Models, JHEP 09 (2009) 038 [arXiv:0906.1461] [INSPIRE].

[30] R. Alonso, G. Isidori, L. Merlo, L.A. Muñoz and E. Nardi, Minimal flavour violation extensions of the seesaw, JHEP 06 (2011) 037 [arXiv:1103.5461] [INSPIRE].

[31] P. Minkowski, $\mu \rightarrow e \gamma$ at a Rate of One Out of $10^{9}$ Muon Decays?, Phys. Lett. B 67 (1977) 421 [INSPIRE].

[32] R.N. Mohapatra and G. Senjanović, Neutrino Mass and Spontaneous Parity Violation, Phys. Rev. Lett. 44 (1980) 912 [INSPIRE].

[33] T. Yanagida, Horizontal gauge symmetry and masses of neutrinos, in SEESAW 25. Proceedings of the International Conference on the Seesaw Mechanism, Institut Henri Poincaré, Paris, June 10-11 2004, World Scientific (2005), pp. 261-264.

[34] M. Gell-Mann, P. Ramond and R. Slansky, Complex Spinors and Unified Theories, Conf. Proc. C 790927 (1979) 315 [arXiv:1306.4669] [inSPIRE].

[35] S. Weinberg, Baryon and Lepton Nonconserving Processes, Phys. Rev. Lett. 43 (1979) 1566 [INSPIRE].

[36] M. Blennow and E. Fernandez-Martinez, Parametrization of Seesaw Models and Light Sterile Neutrinos, Phys. Lett. B 704 (2011) 223 [arXiv:1107.3992] [INSPIRE].

[37] LHCb collaboration, Measurement of Form-Factor-Independent Observables in the Decay $B^{0} \rightarrow K^{* 0} \mu^{+} \mu^{-}$, Phys. Rev. Lett. 111 (2013) 191801 [arXiv:1308.1707] [INSPIRE].

[38] LHCb collaboration, Test of lepton universality using $B^{+} \rightarrow K^{+} \ell^{+} \ell^{-}$decays, Phys. Rev. Lett. 113 (2014) 151601 [arXiv: 1406.6482] [INSPIRE].

[39] E. Fernandez-Martinez, J. Hernandez-Garcia and J. Lopez-Pavon, Global constraints on heavy neutrino mixing, JHEP 08 (2016) 033 [arXiv: 1605.08774] [INSPIRE].

[40] S. Antusch and O. Fischer, Non-unitarity of the leptonic mixing matrix: Present bounds and future sensitivities, JHEP 10 (2014) 094 [arXiv: 1407.6607] [INSPIRE].

[41] L3 collaboration, P. Achard et al., Search for heavy neutral and charged leptons in $e^{+} e^{-}$ annihilation at LEP, Phys. Lett. B 517 (2001) 75 [hep-ex/0107015] [INSPIRE].

[42] ATLAS collaboration, Search for supersymmetry with two and three leptons and missing transverse momentum in the final state at $\sqrt{s}=13 \mathrm{TeV}$ with the ATLAS detector, ATLAS-CONF-2016-096 (2016) [INSPIRE].

[43] E. Eichten, K.D. Lane and M.E. Peskin, New Tests for Quark and Lepton Substructure, Phys. Rev. Lett. 50 (1983) 811 [INSPIRE].

[44] DELPhi, OPAL, LEP Electroweak, ALEPH and L3 collaborations, S. Schael et al., Electroweak Measurements in Electron-Positron Collisions at W-Boson-Pair Energies at LEP, Phys. Rept. 532 (2013) 119 [arXiv:1302.3415] [INSPIRE]. 
[45] R. Jackiw and S. Weinberg, Weak interaction corrections to the muon magnetic moment and to muonic atom energy levels, Phys. Rev. D 5 (1972) 2396 [INSPIRE].

[46] R.N. Mohapatra, Mechanism for Understanding Small Neutrino Mass in Superstring Theories, Phys. Rev. Lett. 56 (1986) 561 [InSPIRE].

[47] R.N. Mohapatra and J.W.F. Valle, Neutrino Mass and Baryon Number Nonconservation in Superstring Models, Phys. Rev. D 34 (1986) 1642 [InSPIRE].

[48] J. Bernabéu, A. Santamaria, J. Vidal, A. Mendez and J.W.F. Valle, Lepton Flavor Nonconservation at High-Energies in a Superstring Inspired Standard Model, Phys. Lett. B 187 (1987) 303 [INSPIRE].

[49] G.C. Branco, W. Grimus and L. Lavoura, The Seesaw Mechanism in the Presence of a Conserved Lepton Number, Nucl. Phys. B 312 (1989) 492 [INSPIRE].

[50] J. Kersten and A.Y. Smirnov, Right-Handed Neutrinos at CERN LHC and the Mechanism of Neutrino Mass Generation, Phys. Rev. D 76 (2007) 073005 [arXiv:0705.3221] [InSPIRE].

[51] A. Abada, C. Biggio, F. Bonnet, M.B. Gavela and T. Hambye, Low energy effects of neutrino masses, JHEP 12 (2007) 061 [arXiv:0707.4058] [INSPIRE].

[52] J.A. Casas and A. Ibarra, Oscillating neutrinos and $\mu \rightarrow e, \gamma$, Nucl. Phys. B 618 (2001) 171 [hep-ph/0103065] [INSPIRE].

[53] Particle Data Group collaboration, J. Beringer et al., Review of Particle Physics (RPP), Phys. Rev. D 86 (2012) 010001 [inSPIRE].

[54] W. Altmannshofer, S. Gori, M. Pospelov and I. Yavin, Neutrino Trident Production: A Powerful Probe of New Physics with Neutrino Beams, Phys. Rev. Lett. 113 (2014) 091801 [arXiv: 1406.2332] [INSPIRE].

[55] CCFR collaboration, S.R. Mishra et al., Neutrino tridents and W Z interference, Phys. Rev. Lett. 66 (1991) 3117 [INSPIRE].

[56] CHARM-II collaboration, D. Geiregat et al., First observation of neutrino trident production, Phys. Lett. B 245 (1990) 271 [INSPIRE].

[57] R.E. Shrock, New Tests For and Bounds On, Neutrino Masses and Lepton Mixing, Phys. Lett. B 96 (1980) 159 [INSPIRE].

[58] R.E. Shrock, General Theory of Weak Leptonic and Semileptonic Decays. 1. Leptonic Pseudoscalar Meson Decays, with Associated Tests For and Bounds on, Neutrino Masses and Lepton Mixing, Phys. Rev. D 24 (1981) 1232 [inSPIRE].

[59] R.E. Shrock, General Theory of Weak Processes Involving Neutrinos. 2. Pure Leptonic Decays, Phys. Rev. D 24 (1981) 1275 [inSPIRE].

[60] P. Langacker and D. London, Mixing Between Ordinary and Exotic Fermions, Phys. Rev. D 38 (1988) 886 [INSPIRE].

[61] J. Schechter and J.W.F. Valle, Neutrino Masses in $\mathrm{SU}(2) \times \mathrm{U}(1)$ Theories, Phys. Rev. D 22 (1980) 2227 [INSPIRE].

[62] S.M. Bilenky and C. Giunti, Seesaw type mixing and $\nu_{\mu} \rightarrow \nu_{\tau}$ oscillations, Phys. Lett. B 300 (1993) 137 [hep-ph/9211269] [INSPIRE].

[63] E. Nardi, E. Roulet and D. Tommasini, Limits on neutrino mixing with new heavy particles, Phys. Lett. B 327 (1994) 319 [hep-ph/9402224] [INSPIRE]. 
[64] D. Tommasini, G. Barenboim, J. Bernabéu and C. Jarlskog, Nondecoupling of heavy neutrinos and lepton flavor violation, Nucl. Phys. B 444 (1995) 451 [hep-ph/9503228] [INSPIRE].

[65] S. Bergmann and A. Kagan, Z-induced FCNC's and their effects on neutrino oscillations, Nucl. Phys. B 538 (1999) 368 [hep-ph/9803305] [inSPIRE].

[66] W. Loinaz, N. Okamura, T. Takeuchi and L.C.R. Wijewardhana, The NuTeV anomaly, neutrino mixing and a heavy Higgs boson, Phys. Rev. D 67 (2003) 073012 [hep-ph/0210193] [INSPIRE].

[67] W. Loinaz, N. Okamura, S. Rayyan, T. Takeuchi and L.C.R. Wijewardhana, Quark lepton unification and lepton flavor nonconservation from a TeV scale seesaw neutrino mass texture, Phys. Rev. D 68 (2003) 073001 [hep-ph/0304004] [INSPIRE].

[68] W. Loinaz, N. Okamura, S. Rayyan, T. Takeuchi and L.C.R. Wijewardhana, The NuTeV anomaly, lepton universality and nonuniversal neutrino gauge couplings, Phys. Rev. D 70 (2004) 113004 [hep-ph/0403306] [INSPIRE].

[69] S. Antusch, C. Biggio, E. Fernandez-Martinez, M.B. Gavela and J. Lopez-Pavon, Unitarity of the Leptonic Mixing Matrix, JHEP 10 (2006) 084 [hep-ph/0607020] [INSPIRE].

[70] S. Antusch, J.P. Baumann and E. Fernandez-Martinez, Non-Standard Neutrino Interactions with Matter from Physics Beyond the Standard Model, Nucl. Phys. B 810 (2009) 369 [arXiv: 0807.1003] [INSPIRE].

[71] C. Biggio, The Contribution of fermionic seesaws to the anomalous magnetic moment of leptons, Phys. Lett. B 668 (2008) 378 [arXiv:0806.2558] [INSPIRE].

[72] R. Alonso, M. Dhen, M.B. Gavela and T. Hambye, Muon conversion to electron in nuclei in type-I seesaw models, JHEP 01 (2013) 118 [arXiv:1209.2679] [INSPIRE].

[73] A. Abada, D. Das, A.M. Teixeira, A. Vicente and C. Weiland, Tree-level lepton universality violation in the presence of sterile neutrinos: impact for $R_{K}$ and $R_{\pi}$, JHEP 02 (2013) 048 [arXiv: 1211.3052] [INSPIRE].

[74] E. Akhmedov, A. Kartavtsev, M. Lindner, L. Michaels and J. Smirnov, Improving Electro-Weak Fits with TeV-scale Sterile Neutrinos, JHEP 05 (2013) 081 [arXiv:1302.1872] [INSPIRE].

[75] L. Basso, O. Fischer and J.J. van der Bij, Precision tests of unitarity in leptonic mixing, Europhys. Lett. 105 (2014) 11001 [arXiv:1310.2057] [INSPIRE].

[76] A. Abada, A.M. Teixeira, A. Vicente and C. Weiland, Sterile neutrinos in leptonic and semileptonic decays, JHEP 02 (2014) 091 [arXiv:1311.2830] [INSPIRE].

[77] S. Antusch and O. Fischer, Testing sterile neutrino extensions of the Standard Model at future lepton colliders, JHEP 05 (2015) 053 [arXiv: 1502.05915] [INSPIRE].

[78] A. Abada, V. De Romeri and A.M. Teixeira, Impact of sterile neutrinos on nuclear-assisted cLFV processes, JHEP 02 (2016) 083 [arXiv: 1510.06657] [INSPIRE].

[79] E. Fernandez-Martinez, J. Hernandez-Garcia, J. Lopez-Pavon and M. Lucente, Loop level constraints on Seesaw neutrino mixing, JHEP 10 (2015) 130 [arXiv:1508.03051] [INSPIRE].

[80] A. Abada and T. Toma, Electric Dipole Moments of Charged Leptons with Sterile Fermions, JHEP 02 (2016) 174 [arXiv: 1511.03265] [INSPIRE]. 
[81] A. Abada and T. Toma, Electron electric dipole moment in Inverse Seesaw models, JHEP 08 (2016) 079 [arXiv: 1605. 07643] [INSPIRE].

[82] T2K collaboration, K. Abe et al., Measurements of neutrino oscillation in appearance and disappearance channels by the T2K experiment with $6.6 \times 10^{20}$ protons on target, Phys. Rev. D 91 (2015) 072010 [arXiv:1502.01550] [INSPIRE].

[83] NOvA collaboration, P. Adamson et al., First measurement of electron neutrino appearance in NOvA, Phys. Rev. Lett. 116 (2016) 151806 [arXiv:1601.05022] [INSPIRE]. 